\title{
Augmenting Scientific Papers with Just-in-Time, Position-Sensitive Definitions of Terms and Symbols
}

\author{
Andrew Head \\ andrewhead@berkeley.edu \\ UC Berkeley
}

\author{
Kyle Lo \\ kylel@allenai.org \\ Allen Institute for AI
}

\author{
Dongyeop Kang \\ dongyeopk@berkeley.edu \\ UC Berkeley
}

\author{
Raymond Fok \\ rayfok@cs.washington.edu \\ University of Washington
}

\author{
Sam Skjonsberg \\ sams@allenai.org \\ Allen Institute for AI
}

\author{
Daniel S. Weld \\ danw@allenai.org \\ Allen Institute for AI
}

University of Washington

\author{
Marti A. Hearst \\ hearst@berkeley.edu \\ UC Berkeley
}

\begin{abstract}
Despite the central importance of research papers to scientific progress, they can be difficult to read. Comprehension is often stymied when the information needed to understand a passage resides somewhere else-in another section, or in another paper. In this work, we envision how interfaces can bring definitions of technical terms and symbols to readers when and where they need them most. We introduce ScholarPhi, an augmented reading interface with four novel features: (1) tooltips that surface position-sensitive definitions from elsewhere in a paper, (2) a filter over the paper that "declutters" it to reveal how the term or symbol is used across the paper, (3) automatic equation diagrams that expose multiple definitions in parallel, and (4) an automatically generated glossary of important terms and symbols. A usability study showed that the tool helps researchers of all experience levels read papers. Furthermore, researchers were eager to have ScholarPhi's definitions available to support their everyday reading.
\end{abstract}

\section{CCS CONCEPTS}

- Human-centered computing $\rightarrow$ Interactive systems and tools.

\section{KEYWORDS}

interactive documents,reading interfaces,scientific papers, definitions, nonce words

\section{ACM Reference Format:}

Andrew Head, Kyle Lo, Dongyeop Kang, Raymond Fok, Sam Skjonsberg, Daniel S. Weld, and Marti A. Hearst. 2021. Augmenting Scientific Papers with Just-in-Time, Position-Sensitive Definitions of Terms and Symbols. In CHI Conference on Human Factors in Computing Systems (CHI '21), May 8-13, 2021, Yokohama, Japan. ACM, New York, NY, USA, 18 pages. https://doi.org/ 10.1145/3411764.3445648

\section{INTRODUCTION}

Researchers are charged with keeping on top of immense, rapidlychanging literatures. Naturally, then, reading constitutes a major

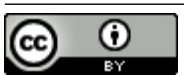

This work is licensed under a Creative Commons Attribution International 4.0 License.

CHI '21, May 8-13, 2021, Yokohama, Japan

(C) 2021 Copyright held by the owner/author(s)

ACM ISBN 978-1-4503-8096-6/21/05.

https://doi.org/10.1145/3411764.3445648

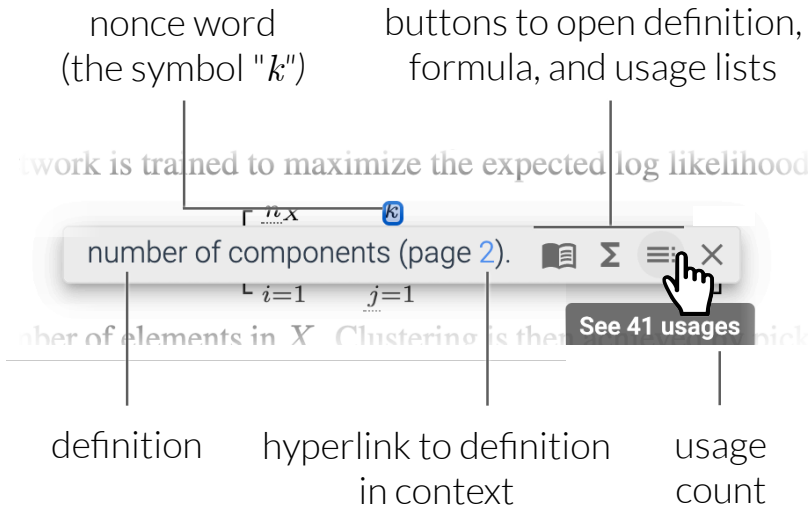

Figure 1: ScholarPhi helps readers understand nonce wordsunique technical terms and symbols-defined within scientific papers. When a reader comes across a nonce word that they do not understand, ScholarPhi lets them click the word to view a position-sensitive definition in a compact tooltip. The tooltip lets the reader jump to the definition in context. It also lets them open lists of prose definitions, defining formulae, and usages of the word. ScholarPhi augments the reading experience with this and a host of other features (see Section 4) to assist readers.

part of a researcher's everyday work. Senior researchers, such as faculty members, spend over one hundred hours a year reading the literature, consuming over one hundred papers annually [97]. And despite the formidable background knowledge that a researcher gains over the course of their career, they will still often find that papers are prohibitively difficult to read.

As they read, a researcher is constantly trying to fit the information they find into schemas of their prior knowledge, but the success of this assimilation is by no means guaranteed [7]. A researcher may struggle to understand a paper due to gaps in their own knowledge, or due to the intrinsic difficulty of reading a specific paper [7]. Reading is made all the more challenging by the fact that scholars increasingly read selectively, looking for specific information by skimming and scanning [34, 70, 98].

We are motivated by the question: Can a novel interface improve the reading experience by reducing distractions that interrupt the reading flow? This work takes a measured step to address the general design question by focusing on the specific case of helping 


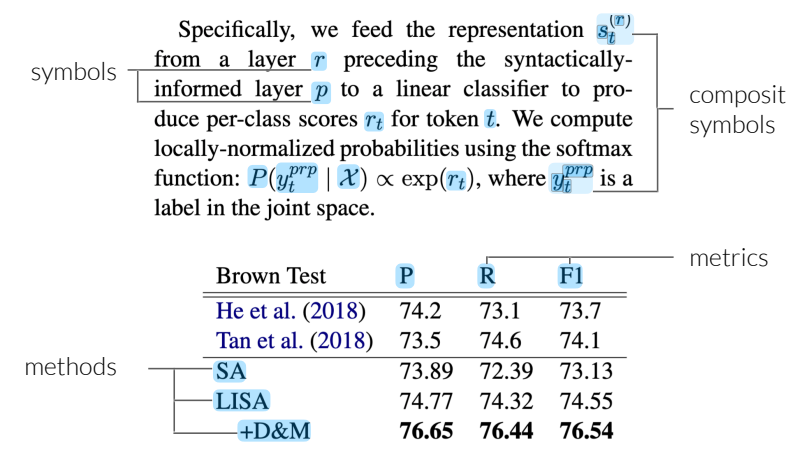

Figure 2: One challenge to reading a paper is making sense of the hundreds of nonce words within them. Nonce words, like the symbols, abbreviations, and terms shown above, are defined within a paper for use within that paper. As such, a reader cannot know what they mean ahead of time. Quintessential examples of nonce words in the computer science literature are mathematical symbols, and abbreviations for metrics, algorithms, and datasets. The excerpts above are from Strubell et al.'s Linguistically-informed self-attention for semantic role labeling [93].

readers understand cryptic technical terms and symbols defined within a paper, which are called "nonce words" in the field of linguistics. Formally, a nonce word is a word that is coined for a particular use, which is unlikely to become a permanent part of the vocabulary [66]. Because a nonce word is localized to a specific paper, a reader cannot know precisely what it means when they start reading the paper. Because it is only intended for use within a single paper, it is likely to be defined somewhere within that same paper, but finding that definition may require significant effort by the reader. By their nature, nonce words are an interesting focus for augmenting reading tools because readers will have questions about them, and those questions will be answerable (exclusively by) searching the text that contains them.

Two aspects of nonce words constrain the design of any reading application that is built to define them. First, they are numerous: a paper can contain hundreds of them. Indeed, a single passage or table may contain a dozen terms closely packed together (see Figure 2). In such settings a reader is likely to have demands on their working memory and may also want to see definitions for multiple nonce words in the same vicinity.

Second, nonce words are sometimes assigned multiple definitions within the same paper. One example is a symbol like $k$, which over the course of a single paper may variously stand for a dummy variable in a summation operation, the number of components in a mixture of Gaussian models, and the number of clusters output by a clustering algorithm (see the scenario in Section 4). These two aspects of nonce words raise the question of whether conventional solutions for showing definitions of terms (e.g., the electronic glossaries explored in second-language learning research $[13,104]$ or Wikipedia's page previews [68]) also suit a researcher who is puzzling their way through dense, cryptic, ambiguous notation.

In this work, we introduce ScholarPhi, a tool that helps readers efficiently access definitions of nonce words in scientific papers.
The larger vision of ScholarPhi is to help scientists more easily read papers by linking relevant information to its location of use. We envision the tool eventually providing access to the contents of cited papers, and definitions external to the paper. The current paper focuses on one portion of this problem: the design and evaluation of interfaces for understanding nonce words.

This paper begins with a formative study of nine readers as they read a scientific text of their own choice (Section 3.1). Most readers expressed confusion at nonce words in their texts. Many readers were reluctant to look up what the words meant, given the anticipated cost of doing so. This inspired the subsequent design of a tool that could have answered those readers' questions while reducing friction so that readers would actually use the tool.

We then describe design motivations for a new reading interface (Section 3.3) that are grounded in insights from four pilot studies with early prototypes, conducted with 24 researchers. Key insights from the research include the importance of tailoring definitions to the passage where a reader seeks to understand a nonce word, and the competing goals of providing scent (i.e., visual cues [76]) of what is defined without distracting from a reading task that is already cognitively demanding on its own.

Building on the motivations found in the pilot research, a user interface is presented (Section 4). The basic design of ScholarPhi is one of an interactive hypertext interface. A reader's paper is augmented with subtle hyperlinks indicating which nonce words can be clicked in order to access definition information. Readers can click nonce words to access definitions for those words in a compact tooltip (Figure 1). These definitions are position-sensitivethat is, if there are multiple definitions of a nonce word in the text, ScholarPhi uses the heuristic of showing readers the most recent definition that appears before the selected usage of the word. Definitions are also linked to the passage they were extracted from: a reader can click on a hyperlink next to the definition to jump to where it appears in the paper. In addition to definitions, the tooltip makes available a list of all usages of the nonce word throughout the text, as well as a special view of formulae that include the word.

Beyond these basic affordances, ScholarPhi provides a suite of features, each of which provides readers with efficient yet nonintrusive methods for accessing information about nonce words. First, ScholarPhi provides efficient, precise selection mechanics for selecting mathematical symbols and their sub-symbols through single clicks, rather than error-prone text selections (Section 4.1). Second, ScholarPhi provides a novel filter over the paper called "declutter" that helps a reader search for information about a nonce word by low-lighting all sentences in the paper that do not include that word (Section 4.2). Third, ScholarPhi generates equation diagrams and overlays them on top of display equations, affixing labels to all symbols and sub-symbols in the equation for which definitions are available (Section 4.3). The final feature is a priming glossary comprising definitions of all nonce words that appear in a paper, prepended to the start of the document (Section 4.4).

The emphasis in the design of each of these features is on acknowledging the inherent complexity of the setting of scientific papers, and hence designing features for looking up definitions that are easy to invoke and minimally distracting.

To enable these features, new methods were introduced for analyzing scientific papers in order to make nonce words interactive. 
A paper processing pipeline was built that automatically segments equations into symbols and their sub-symbols, detects all usages for a nonce word, and detects precise bounding box locations of nonce words so that they may be clicked. The implementation of the pipeline is described in Section 5. The suitability of contemporary definition extraction algorithms is discussed, highlighting a need for improvements to technologies for definition extraction.

This work concludes with a controlled usability study with twenty-seven researchers (Section 6). Researchers were observed as they used three versions of ScholarPhi-one with all the features described above, one with only the "declutter" feature, and one that behaved exactly like a standard, un-augmented PDF reader.

When readers had access to ScholarPhi's features, they could answer questions about a scientific paper in significantly less time, while viewing significantly less of the paper in order to come to an answer. They reported that they found it easier to answer questions about the paper, and were more confident about their answers with ScholarPhi. Researchers were also observed as they used ScholarPhi for 15 minutes of unstructured reading time. Researchers made use of all of ScholarPhi's features. Feedback was overwhelmingly positive. Most participants expressed interest in using the features "often" or "always" for future papers, with an emphasis on the usefulness of definition tooltips and equation diagrams.

In summary, this work makes four contributions. First, it characterizes the problem of searching for information about nonce words as one of the challenges of reading scientific papers, grounded in a small formative study. Second, it provides design motivations for designing interactive tools that define nonce words, grounded in iterative evaluations of prototypes of a tool. Third, it presents ScholarPhi, an augmented reading interface with a suite of novel features for helping readers understand nonce words in scientific papers. Finally, it provides evidence of the usefulness of the design in searching and reading scientific papers through a controlled study with twenty-seven researchers. ${ }^{1}$

\section{BACKGROUND AND RELATED WORK}

\subsection{How Researchers Read Papers}

Researchers read papers to become aware of foundational ideas and to stay apprised of the latest developments in their field. However, reading papers is difficult. Challenges in reading a paper can come from gaps in a reader's knowledge, or from ideas in the paper that are poorly explained [7]. Papers may be read out-of-order and piecemeal $[7,34,70]$. As a result, a passage of a paper may be read out of context. An additional challenge is assimilating information scattered across one or many documents, a challenge common to the activity of active reading in many domains [2, 72, 94].

Papers that include mathematical content can impose additional demands on a reader. Reading mathematical texts often entails grappling with unfamiliar terminology and notational idioms, which can be particularly challenging for less experienced readers [90]. Selfreports from mathematicians have suggested that the process of reading math involves backtracking as a reader attempts to scaffold their understanding [101], a pattern which has also been observed in eye-tracking studies of reading math $[38,49]$. When attempting

\footnotetext{
${ }^{1}$ An interactive demo, video figure, and source code for this work can be found on the project website at https://scholarphi.org.
}

to understand an equation, readers will look to nearby equations and text for clarifications [49].

While reading papers in physical volumes and print-outs used to be the norm, it is increasingly the case that researchers consult papers in digital reading applications $[54,97]$, particularly for some types of scholarly communication such as conference proceedings [54]. This suggests the value of investing in reading user interfaces that take advantage of the unique interactive potential of digital interfaces to augment the reading experience.

\subsection{Augmented Reading Interfaces}

Since the beginning of human-computer interaction as a discipline, one of the foundational challenges has been equipping knowledge workers with tools that extend their cognition during reading. Vannevar Bush, in his vision of the memex, proposed a system that enabled readers to build trails across the literature, linking passages across related readings in a way that made implicit connections clear [9]. This vision has expressed itself in many forms, from the invention of hypertext [18] to experiments with interactive books [24, 71] and "fluid documents" that can adapt their form and content to elaborate where readers need clarification [11]. In the first decade of the $\mathrm{CHI}$ conference, myriad techniques were proposed to help readers navigate text using social annotations [33], to augment hypertexts with glosses that could dynamically change the layout of the text [108], and to provide navigational affordances that allow readers to see overviews of document content and jump quickly to passages of interest $[28,86]$.

2.2.1 Glossaries, Definitions, and Explanations. Today, many reading and editing tools show dictionary definitions when a reader hovers over or clicks on a word. The Word Wise feature in the Amazon Kindle lets readers view definitions of tricky words in the space between consecutive lines of text [61]. In 2014, Wikipedia began to roll out page previews as a feature that allowed readers to preview the content of a referenced page by hovering over a link to that page. Based on positive usability evaluation results, Wikipedia decided to make the feature a permanent fixture on the site [68]. Recent proceedings of human-computer interaction conferences have introduced prototypes that allow readers to answer their questions about how to use web pages [14], the meaning of cryptic programming syntax [30], hard-to-visualize quantities [37], and unfamiliar words from a second language [62].

2.2.2 Symbol Selection. ScholarPhi uses an advanced symbol selection technique that draws from related work. Zeleznik et al. [107] introduced gestures for a multi-touch display that support the efficient selection of mathematical expressions. Bier et al. [8] designed a technique for rapid selection of entities in text (such as addresses) with a single click. The symbol selection mechanism in ScholarPhi can be seen as a combination of these two features, supporting single-click selection of mathematical expressions, with refinement of the selection to choose specific sub-symbols of that expression via additional clicks. In the future, ScholarPhi may support the efficient selection of many nonce words at once in a passage using fuzzy text selection techniques such as those proposed by Hinckley et al. [35] and Chang et al. [12]. 
2.2.3 Information Highlighting and Fading. ScholarPhi is designed to support the efficiency of visual querying present in contemporary code editors like VSCode [100], in which arbitrary text (i.e., a variable or expression) can be selected, and all other appearances of that same text are instantly highlighted everywhere else in the text. In the design of its lists of definitions and usages, ScholarPhi also draws inspiration from tools such as LiquidText [95], which supports viewing lists of within-text search results side-by-side with the query term highlighted. In its design of the "declutter" filter, ScholarPhi draws on the design of visual filters already present in prototype and production tools. The fading out of content in order to direct a reader's focus to information of interest is a design pattern that has been used in interactive tutorials [44] in which instructions are highlighted while the rest of the user interface is faded, as well as in interactive debugging tools [22, 47].

2.2.4 Readability versus Document Augmentations. On the whole, evidence has supported the usefulness of embedding explanations in texts. In the context of second-language learning, embedded glosses for unfamiliar vocabulary have been shown to lead to vocabulary learning [104], and improved comprehension [96].

That said, in making texts interactive, there is a key tension between assisting the reader and distracting them. On the one hand, studies such as one run by Rott [82] suggest that the best comprehension outcomes can be achieved when all words that have glosses are marked. On the other hand, interactive texts change a reader's behavior. Understandably, readers are more likely to click on words that are visibly interactive [20], leading to what has been called by some "click-happy behavior" [81]. Furthermore, studies of texts augmented with hyperlinks have sometimes shown that these augmentations lead to worse comprehension of the texts, rather than better comprehension [21]. What the evidence suggests overall is that amidst the appeal of interactive reading interfaces, great care must be taken during design to make sure not to introduce features that will ultimately distract readers from the cognitively demanding task of reading.

\subsection{Tools for Reading Scientific Papers}

2.3.1 Links to External Resources. Tools can help researchers read scientific papers in a number of ways. To reduce the need to click away from the paper currently being read, some online journals now allow readers to view metadata by clicking on citations [25, 79, 92]. Experimental tools have been built that augment papers with additional information about cited papers [78], bias in study design [63], and links to external learning resources [40,59]. They have supported interpersonal explanations, allowing peer reviewers [74], collaborators [106], instructors [67], strangers [26], and crowds [39] to annotate and discuss passages of papers. Other approaches to saving the scientist time include tools to support literature search (e.g., $[77,80,109])$, summarize text $[10,87]$, or rewrite passages in simpler language [46].

2.3.2 Links within Papers. Reading interfaces can also assist researchers by helping them navigate to information of interest within a paper. For several years, interfaces for reading PDFs have provided standard affordances for jumping within a paper using hyperlinks. Typesetting software like LTEX can automatically embed clickable links from references to figures, equations, and sections to the content they refer to, and from citations to bibliographies.

Prototype tools have been built to further assist readers in finding passages about topics of interest [28], in jumping between a passage that describes research results to the relevant parts of data tables $[4,45,51]$, and in jumping to passages that answer their natural language questions [110]. Other tools has augmented static figures in papers with animated [29] or interactive [64] overlays.

Of particular relevance to this paper are experimental systems that surface explanations of terms and symbols in scientific papers. Tools have been developed that link from terms to the pages that define them on Wikipedia [1], and which link from key phrases in papers to topic pages where those phrases are defined alongside excerpts about those topics from other papers [89].

2.3.3 Tools for Reading Math. In response to the unique challenges of reading mathematical texts, prototype tools have been designed to expose definitions of math expressions within a text $[3,49,73]$. e-Proofs provide guided tours of proofs, selectively fading parts of the proof that are not currently the focus of the tour [3]. e-Proofs were designed to augment single-page proofs rather than papers. The Planetary system lets readers look up the meanings of operator symbols in external knowledge bases, and reveals simplified versions of equations with details elided [50].

Studies of the e-Proofs system (see $[83,84]$ ) hint at design tensions in tools for reading math. It was found that while readers used the tools of their own accord [83], many features that were introduced to assist readers, such as audio walkthroughs of the content, got in readers' ways [84]. ScholarPhi consolidates and extends features from these prior prototypes, and introduces additional features and affordances, with the goal of helping readers understand mathematical symbols among other nonce words.

\section{DESIGN MOTIVATIONS}

The design of ScholarPhi is motivated by insights from an iterative design process. This section reports insights arising from a formative study, a review of the related work, prototyping efforts, and informal usability studies of early prototypes.

\subsection{Formative Study}

To better understand how the presence of nonce words affects the reading experience, we conducted a small formative study. Nine readers (four graduate students, five undergraduate students, referred to as R1-9 below) participated in an observational study in which they read a scientific text of their own choice. Six participants brought research papers (R1-5, R8); five of these papers were about computer science and one was about architecture. Three participants brought instructional texts on the topics of data science (R6), experimental design (R7), and formal analysis (R9).

Participants were asked to read their text for forty minutes and simultaneously think aloud. Readers reported when they encountered confusing passages of text and described whether they intended to look up information to clarify their confusion. If they chose to look for such clarifying information, they described where they looked and why. Our findings were as follows:

All but one reader expressed confusion at a term used in the text (R1-3, R5-9). In some cases, the confusion was about a term that 
was specific to the scientific discipline of the text (R3, R5-9), such as the terms "diacritic" (R3) or "population parameter" (R6). For papers from computer science, such terms included benchmarks used to test an algorithm (R3) and baselines against which an algorithm of interest was compared (R5).

In other cases, the terms causing confusion came from within the same paper. Authors introduced terms to describe their methods ("symbolic validator" (R1), "backtranslation" (R3)) whose meanings readers could not surmise when viewed apart from their definitions. Authors would invent shorthand for running examples (e.g., a test set of cow images named "cow") that they then referred to by that shorthand (i.e., "cow") in figures, which could be confusing if the reader was reading the text out of order (R5). Texts could also be sprinkled with vague back-references to assumptions (R5), analyses (R6), parameters (R8), and theorems (R9) that readers could not recall. In some cases (R6, R8), readers were not sure whether a term referred to a passage in the current text or in another text.

Mathematical symbols were another source of confusion (R2-4, R6). Readers sometimes simply could not understand the meaning of a symbol (e.g., " $\Theta_{s}$," “ $M$," "p," “ q," “ $x$," " $y$," “ $y_{1}$, , R2-4, R6). In other cases, they wanted information about how a set of symbols were used in combination. For example, R4 scanned the appendix of the research paper they were reading to better understand the meaning of a ratio " $M / N$ " that appeared in one of the equations. Readers also wondered about the values that symbols were assigned (R2, R3, R6). For example, one reader (R2) wondered what value the regularization parameter $\lambda$ was set to when a model was trained. Another reader (R3) wanted to see example data that could be used as inputs $x$ and $y$ to a translation algorithm.

Thus, confusion about terms and symbols (nonce words, in our terminology) was common among the readers in the study. Readers' strategies for resolving this confusion varied based on how important it was that they understood a nonce word. If it seemed important, a reader often attempted to infer meaning from context (R3, R6-9). If they could not surmise the meaning from context, readers would sometimes delay looking up an explanation with the hope that they might find one later in the text (R1, R3, R4, R6-9). A drawback of this approach, described by $\mathrm{R} 1$, is that a reader may reach a point in the text where they lack an understanding of so many important terms that they can no longer understand the text without stopping and searching for explanations.

Eventually, many readers needed to do just that, and stopped reading in order to look up explanations. One participant referred to this as an undesirable "context switch" which takes them out of the "headspace" of understanding a complicated passage (R4). When looking for explanations, five readers looked elsewhere in the same text (R2-4, R8, R9). This entailed backtracking within the text (R3, R4), jumping forward (R2, R4), opening within-text glossaries (R8), and performing within-text search (i.e., "Control-F” search) within the reading application (R9). Those reading instructional texts often consulted external references like web search results (R6, R8), dictionary applications (R7), and Wikipedia (R9). One reader took a proactive approach to reducing the cost of within-paper lookups by assembling glossaries for key symbols in the margins of the text (R4, see Figure 3).

This study indicated that readers of scientific papers, and scientific texts more generally, frequently have questions about nonce

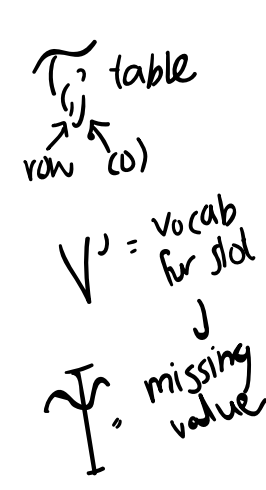

entities, columns correspond to the unique relation types (slots henceforth), and some entries may be missing. An example is shown in Figure 1.

\subsection{Notations and Assumptions}

Let $\mathcal{T}$ denote the KB table described above and $\mathcal{T}_{i, j}$ Cenote the $j$ th slot-value of the $i$ th entity.

$1 \leq i \leq N$ and $1 \leq j \leq$ We let $V^{j}$ denote the vocabulary of each slot, i.e. the set of all distinct values in the $j$-th column. We denote missing values from the table with a special token and write $\mathcal{T}_{i, j}=\Psi . M_{j}=\left\{i: \mathcal{T}_{i, j}=\Psi\right\}$ denotes the set of entities for which the value of slot $j$ is missing. Note that the user may still know the actual value of $\mathcal{T}_{i, j}$, and we assume this lies in $V^{j}$. We do not

Figure 3: When researchers have trouble understanding nonce words, they look up explanations elsewhere. One researcher in the formative study proactively assembled glossaries in the margins of the paper for key symbols (above). The researcher annotated the text with definitions of symbols and miniature equation diagrams (see the annotation for $\mathcal{T}_{i, j}$ ).

words. To answer these questions, readers either infer answers from context, wait for an answer, or look for explanations elsewhere. While readers do look for explanations elsewhere, they try to avoid doing so as it takes them away from the text they are trying to understand. These observations suggest that readers could benefit from interfaces that make explanations of nonce words, and unfamiliar terms more generally, available to them without distracting them from the task of a careful reading.

\subsection{Design Process}

The design of ScholarPhi was refined through an iterative design process lasting twelve months. Our research followed a process similar to research through design [111], consisting of iterative ideation, prototyping, and assessment. This process yielded a multifaceted design space, representing choices that must be made when designing an interactive tool that shows definitions of nonce words.

Design alternatives were identified by reviewing literature reviews on e-glossaries (e.g., [81, 104]) and research and commercial tools (see Section 2), and by brainstorming within our team and with users of early prototypes. The design space appears in Table 1.

Five prototypes were developed and assessed. The last of these prototypes is the tool we call ScholarPhi, and is described in Section 4 and assessed in Section 6. The first four prototypes were designed to evaluate promising design alternatives. Table 1 indicates which features were present in early prototypes, and in the final design.

Each prototype was evaluated in a pilot study of its own:

- Study D (Declutter lens only): 4 researchers (D1-4)

- Study $S$ (Side notes containing definitions, defining formulae, and usages): 4 researchers (S1-4)

- Study $T$ (Tooltips instead of side notes): 9 researchers (T1-9)

- Study $E$ (Equation diagrams and a complex version of tooltip interaction flow): 9 researchers (E1-9).

The first two studies ( $D$ and $S$ ) were observational studies. Participants thought aloud as they used the tool. For the second two 
Definition selection and presentation

\begin{tabular}{|c|c|c|c|c|c|}
\hline \multirow{3}{*}{$\begin{array}{r}\text { Dimension } \\
\text { Source } \\
\text { Selection }\end{array}$} & \multicolumn{5}{|c|}{ Alternatives } \\
\hline & Same section & \multicolumn{2}{|c|}{ Same paper } & \multicolumn{2}{|c|}{ Other papers } \\
\hline & Closest & First & \multicolumn{2}{|c|}{ Most relevant } & All \\
\hline Modality & Text & \multicolumn{2}{|c|}{ Audio } & \multicolumn{2}{|c|}{ Formulae } \\
\hline Appearance & $\begin{array}{l}\text { Document } \\
\text { preview }\end{array}$ & \multicolumn{2}{|c|}{$\begin{array}{c}\begin{array}{c}\text { Reflowable } \\
\text { text }\end{array} \\
\end{array}$} & \multicolumn{2}{|c|}{$\begin{array}{l}\text { None (link } \\
\text { to passage) }\end{array}$} \\
\hline Placement & Margins & $\begin{array}{l}\text { Between } \\
\text { lines }\end{array}$ & $\begin{array}{r}\text { Sideb } \\
\text { foot }\end{array}$ & \multicolumn{2}{|c|}{ Tooltips } \\
\hline $\begin{array}{r}\text { Error } \\
\text { recovery }\end{array}$ & $\begin{array}{ll}\text { Link to } & \text { E } \\
\text { context } & \text { u }\end{array}$ & $\begin{array}{l}\text { xppose } \\
\text { Isages }\end{array}$ & $\begin{array}{r}\text { Convey } \\
\text { uncertain }\end{array}$ & & $\begin{array}{l}\text { ltiple } \\
\text { itions }\end{array}$ \\
\hline
\end{tabular}

\begin{tabular}{|c|c|c|c|c|}
\hline \multicolumn{5}{|c|}{ Access to definitions } \\
\hline Dimension & \multicolumn{4}{|c|}{ Alternatives } \\
\hline $\begin{array}{r}\text { Type of } \\
\text { nonce word }\end{array}$ & Protologism & Symbol & \multicolumn{2}{|c|}{ Abbreviation } \\
\hline Scent & $\begin{array}{l}\text { All nonce } \\
\text { words }\end{array}$ & \multicolumn{2}{|c|}{$\begin{array}{c}\text { Only nonce words } \\
\text { with definitions }\end{array}$} & $\begin{array}{r}\text { No } \\
\text { scent }\end{array}$ \\
\hline $\begin{array}{r}\text { Subsymbol } \\
\text { selection }\end{array}$ & Top-down & Bottom-up & Fuzzy & Lasso \\
\hline $\begin{array}{r}\text { Revealing } \\
\text { usages }\end{array}$ & \multicolumn{4}{|c|}{ Lists Highlighting Lowlight other content } \\
\hline
\end{tabular}

Table 1: Design alternatives for applications that reveal definitions of terms and symbols. Alternatives tested in our early prototypes are highlighted in gray. Those selected for inclusion in our final design are circled with a solid border.

studies ( $T$ and $E$ ), participants read on their own, participated in a 30-minute focus group discussion, and filled out a questionnaire about their experience. Seven participants in these last two studies (T1-3, E1-4) participated in a 15-minute follow-up interview. In each study, participants read a different scientific paper. Two researchers (S2, S3) participated in multiple studies.

One author analyzed transcripts from all studies following a qualitative approach. This yielded the following seven design motivations for designing effective interfaces for providing in-situ definitions within scientific texts.

\subsection{Design Motivations}

M1. Tailor definitions to the location of appearance. The same nonce word can have multiple conflicting definitions throughout a paper. For example, in the paper used as stimulus in the formal study [93], the symbol $T$ took on multiple distinct senses including referring to the dimensionality of a vector $x_{t}$, being part of a composite symbol $T^{(j)}$ used to refer to a layer in a neural network, and being used as the matrix transposition operator in several display equations. When readers used a prototype that showed definitions of all of these senses in a list, they wanted to know which ones were the most appropriate to the passage that they were reading (S1-3).
Readers requested that the tool show the definitions appropriate to the place where they asked for them (S1). They also asked to see the context surrounding a definition (S2, S3).

A related principle is eliminating redundant definitions. If a reader selected a nonce word within a passage where it was being defined, they did not wish to see a tooltip containing the definition sentence they were already reading $(\mathrm{S} 1, \mathrm{~T} 9)$.

M2. Connect readers to definitions in context. Four readers requested the ability to jump from a definition to the passage where it appeared in the paper (S1-3, T5, T6). This would help them judge the relevance of the definition (S1-3) and assess what they suspected may be incorrectly extracted definitions (T5).

M3. Consolidate information. While the information that explains a nonce word can be scattered across a paper, readers want explanations that consolidate all of that information in one compact, concise passage. When they clicked on a composite symbol, they wanted to see explanations of each sub-symbol that made up the symbol (E2, E4). They also expected the interface to be able to gather explanations for semantically similar symbols that differed in their surface features, such as showing a definition for "PMA $(\cdot)$ " that was extracted for the function "PMA $(X)$ " (E1).

M4. Provide scent. In all prototypes, nonce words were marked with a light dotted underline. Readers appreciated that the underlines provided scent of which words they could click to see definitions (S2-4). Participants did not turn off this feature, although they were provided with this option in later versions of the design.

M5. Minimize occlusion. In two prototypes, tooltips were packed with definitions, defining formulae, and usages for symbols. Readers reported that these tooltips occluded text that they wished to see (T4, T6, E7) without providing much value beyond the first definition (T1, T4-6). Still, some readers desired tooltips as opposed to side notes, as it allowed them to view definitions without losing their place in the text (E3, E4). The current prototype attempts to balance these conflicting needs by providing a compact tooltip that contains only the most recent definition of a nonce word and a few small buttons for accessing lists of definitions, defining formulae, and usages. A tooltip for a nonce word can be hidden by clicking on a "close" button within the tooltip.

M6. Minimize distractions. The user interface was revised several times to remove features that, while originally envisioned as being helpful, distracted from the reading task. One reader aptly described, "I was trying to pay more attention to the paper than the tool and the paper requires a lot of overhead to understand. So I didn't have much left over for the tool" (E1). One prototype used several highlighting colors to indicate appearances, usages, and definitions of a selected nonce word; however, this added visual clutter that was hard to understand (E3). The current prototype uses a single static highlight color. Readers were asked across multiple studies whether they found underlines beneath the nonce words distracting. They repeatedly reported that they did not (S2-4, T5, T7). However, one reader did request the ability to turn them off (E1), which has been included in all recent prototypes of the interface.

M7. Support error recovery. The systems that are used to detect definitions in scientific papers are prone to error; user interfaces that 
build off of this technology must take this into account. Drawing on guidelines from the literature on interacting with intelligent interfaces when there are errors (synthesized by Wright et al. [103]), the ScholarPhi interface makes use of the following guidelines: provide paths forward, and support efficient dismissal.

Other guidelines that appear in the literature, such as promoting user corrections of errors and displaying auto-detected errors, may impose distractions while reading, and so were not incorporated into the current version of the interface, although future work may determine that these approaches are helpful.

\section{USER INTERFACE}

We illustrate the experience using ScholarPhi through a set of four scenarios, where a reader wishes to know the meaning of a specific nonce word. Each scenario is chosen so that one of ScholarPhi's features is uniquely well-suited to the reader's task.

To explain the design decisions underlying a feature, we refer back to findings from the formative research. Specifically, we note whenever a design choice was informed by one of the design motivations M1-7 that were introduced in Section 3.3. ${ }^{2}$

\subsection{Definition Tooltips}

When a reader wants to know the meaning of a nonce word, ScholarPhi lets them look up the meaning by clicking the nonce word. This reveals a definition tooltip (see Figure 1).

Definition tooltips appear directly beneath the selected nonce word. This placement is intentional. By placing the definition beneath the word, as opposed to placing it in a document margin or a glossary elsewhere in the text, a reader need not divert their gaze from the text. In this way, the tooltip placement is chosen to minimize distraction (M6). Furthermore, to avoid occluding the text (M5), tooltips are compact. Their dimensions never exceed half the page width, nor are they permitted to be longer than four lines tall.

If there are multiple definitions of a nonce word available within the paper, ScholarPhi shows the definition that it infers as being most relevant to the context. Specifically, it uses a heuristic of showing the definition that appeared most recently before that appearance of the word. This reduces mental effort that seeing multiple definitions over the nonce word would incur (M1) and reduces the amount of text occluded by the tooltip (M5).

For instance, in the following passage from Lee et al. [55], $k$ initially refers to an index of a component in a mixture of Gaussians.

$$
\begin{aligned}
& \mathrm{SAB}_{L}(X), H_{\theta}=\mathrm{PMA}_{k}\left(H_{X}\right),\left(\operatorname{logit} \pi_{j}, \theta_{j}\right)_{j=1}^{k}=\mathrm{rFF} \\
& \text { ixing coefficient and } \theta_{j}=\left(\mu_{j}, \sigma_{j}^{2}\right) \text { are the mean and variar } \\
& \text { network is trained to maximize the expected log likelihood } \\
& \qquad \Gamma^{n_{X}} \text { number of components (page 2). 目 } \Sigma \equiv: \times
\end{aligned}
$$

However, in a later passage, $k$ is given an entirely different meaning-a parameter that controls the number of clusters output

\footnotetext{
${ }^{2}$ The papers in these scenarios are recent computer science papers by Lee et al. [55] and Strubell et al. [93]. The latter paper, of which large passages are shown, is published in the EMNLP 2018 proceedings under a Creative Commons ShareAlike-4.0 License.
}

by a clustering algorithm. When the reader opens a tooltip in this other passage, they again see the appropriate definition.

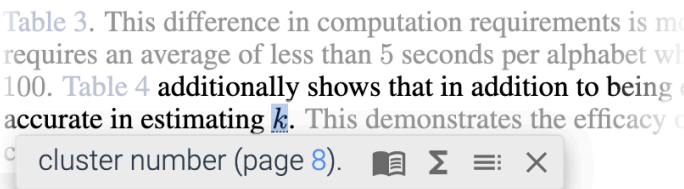

After seeing a definition in the tooltip, a reader may want more information about the nonce word. For instance, they may want to know whether the authors recommended that a specific number of $k$ components be used in the mixture of Gaussians. To help the reader answer questions like this, ScholarPhi connects the reader to definitions in context (M2). The reader can view the definition in context by clicking the hyperlink next to the definition (e.g., "page 2"). ScholarPhi scrolls the paper to the definition, highlighting the sentence that the definition came from:

ample using ST for amortized inference for a MoG. A dataset $X$ kelihood of a $k$ component MoG, and a ST is used to output the

When the reader has finished consulting the highlighted passage, they can click their web browser's "Back" button to return to the definition tooltip at their previous position in the document.

Lists of usages. A reader can also look for more information about a nonce word by reviewing the usages of the word. To connect a reader with these usages, the definition tooltip provides three buttons. The buttons let a reader open lists of all prose definitions of the word, all defining formulae (i.e., formulae in which the nonce word appears on the left-hand side of an assignment), and all usages (i.e., passages that refer to the nonce word). Together, the buttons provide a way for readers to access a consolidated collection of everything that ScholarPhi knows about a nonce word (M3).

When a reader clicks a button, the corresponding list opens in a dedicated sidebar, rather than in the tooltip, to avoid occluding the text (M5). Each usage in the list comprises one sentence referring to the nonce word and a link to the sentence where it appears in the paper (M2). To help readers evaluate the relevance of a usage among dense text and equations, the nonce word is highlighted.

\section{Usages}

Showing 3 usages of the selected symbols.

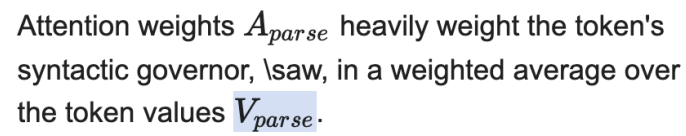

See in context on page $\underline{3}$.

The tooltip buttons for opening usage lists provide scent to help a reader understand how a nonce word is defined and used (M4). By 
hovering over a button, the reader can see how many definitions, defining formulae, or usages there are for a nonce word. A button is disabled if no definitions, defining formulae, or usages exist.

To avoid disorienting the reader, a tooltip always makes the same information available to a reader in the same layout: buttons for lists of definitions, defining formulae, and usages, as well as a definition if one is available. If a tooltip is opened for a nonce word within the sentence where the word is defined, the definition tooltip reports, "Defined here." This way, tooltips do not distract the reader from the text with a definition they have already seen, or are about to see (M6). If no definition exists for the nonce word, then the three buttons to access the usage lists are still shown, but those with no information behind them are grayed out.

Scent. While some nonce words are defined in a paper, others are not. Authors may assume the meaning of a nonce word is implicit or they may simply forget to define it. ScholarPhi provides visual scent [76] to help readers determine whether they'll find a definition for a nonce word before they click on it (M4). This visual scent is provided in the form of a subtle dotted gray underline beneath the nonce word. For instance, in the following passage, readers can open definition tooltips for any of the underlined nonce words, "CoNLL-2005," "SRL," or "LISA."

\section{In experiments on CoNLL-2005 SRL, LISA}

So that it does not divert a user's attention from the text needlessly (M6), ScholarPhi assumes that a reader will not want to view a nonce word in a sentence that defines it, and so does not underline these occurrences. The rules for underlining symbols are more nuanced. Papers can contain composite symbols where certain sub-symbols (e.g., subscripts or superscripts) are defined, but the composite symbol as a whole is not. In such a case, ScholarPhi highlights sub-symbols for which definitions are available. In the passage below, ScholarPhi highlights symbols to indicate that definitions are available for " $t$," " $X$," and " $r_{t}$." Because the composite

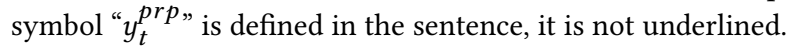

locally-normalized probabilities using the softmax function: $P\left(y_{t}^{p r p} \mid \mathcal{X}\right) \propto \exp \left(r_{t}\right)$, where $y_{t}^{\text {prp }}$ is a label in the joint space.

Symbol selection. In a conventional interface for reading papers, one challenge to searching for information about a symbol is simply selecting the symbol. Because the text for a symbol is often split across multiple baselines (i.e., in subscripts or superscripts), conventional text selection mechanisms may fail to select precisely those characters that belong to the symbol. To reduce the cost of accessing explanations, ScholarPhi supports efficient selection of symbols. Symbols can be selected by clicking them once (steps "1" and "2" below). Once a symbol is selected, all sub-symbols that belong to it are highlighted and can be selected with a click (" 3 ”).

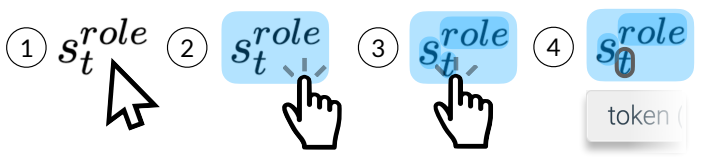

By helping readers rapidly select sub-symbols, it is hoped that ScholarPhi lets readers understand the meaning of a composite symbol in terms of the meanings of its parts (M3).

\subsection{Declutter}

To help readers quickly find information about a nonce word that is scattered across a paper, ScholarPhi provides a novel feature called "decluttering." When a reader selects a nonce word, ScholarPhi "declutters" the paper-by highlighting segments of text that contain matches, and fading out all other sentences-in an effort to help readers scan the paper for usages.

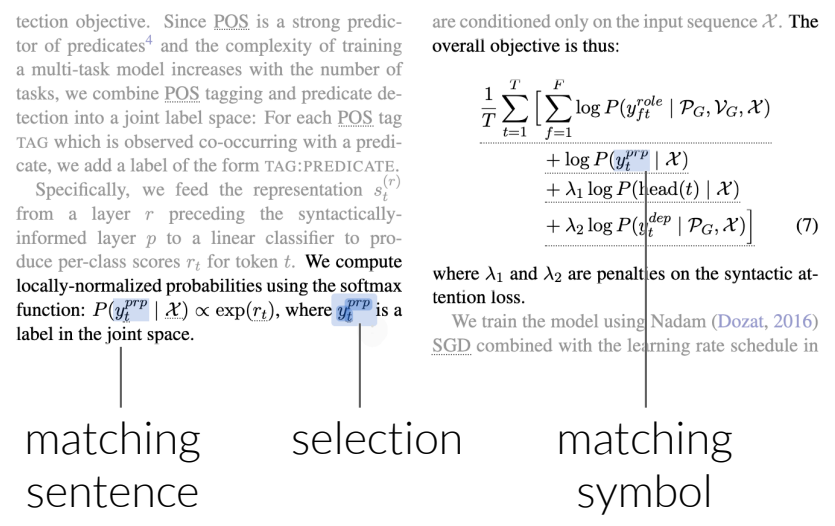

ScholarPhi provides visual scent (M4) of where usages can be found via a conventional search bar. The search bar counts how many times the nonce word appears in the paper, and shows the page number of the usage the reader selected. While readers are expected to navigate a decluttered document by scrolling through it, the search bar also supports navigation between usages with "Next" and "Previous" buttons and arrow key keyboard shortcuts.

Decluttering offers two advantages over the list of usages: it connects readers to definitions in context with a view that is grounded in the text (M2) and it reduces distractions by hiding irrelevant content, rather than showing additional interface widgets (M6). Like the list of usages, decluttering does not occlude text (M5).

\subsection{Equation Diagrams}

Some passages are rife with nonce words. For instance, tables of empirical results are indexed by abbreviations that represent experimental conditions and measurements. Equations contain dozens of symbols. For dense passages like these, readers may desire the ability to consult the definitions for many nonce words at the same time. For display equations in particular (i.e., equations that are shown on their own line separated from the text), ScholarPhi provides the ability to view definitions of all symbols at the same time. To see the definitions of all symbols in a display equation, a reader can click that equation. Definitions are affixed to all symbols simultaneously. 


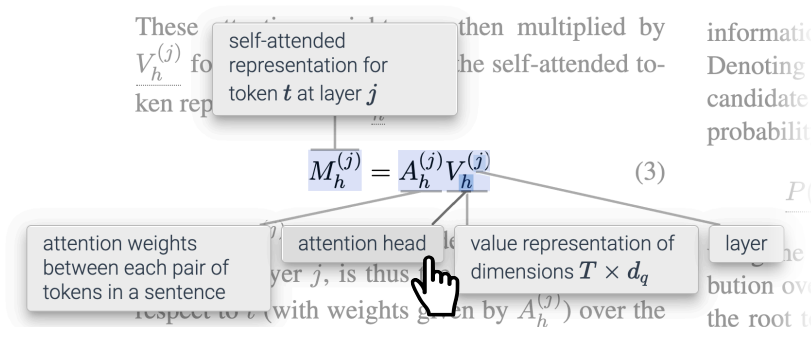

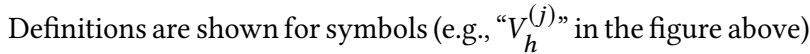
and the sub-symbols they are composed of (" $h$," " $j$ "). Thus, definition information that would otherwise be split across multiple tooltips is consolidated into one place (M3). Like the definitions that appear in tooltips, the definitions for equation diagrams are position-sensitive (M1). By clicking a label for a symbol, a reader can open the definition tooltip for the symbol, providing access through the definition tooltip to the context of the definition (M2).

Brushing and linking connects the definition labels to the symbols; as a reader hovers over a label, the symbol it defines is highlighted with a more saturated color than the other symbols. Leader lines connect the definitions to the symbols. The leader lines connecting definitions to symbols are diagonal, proceeding straight from the definition label to the symbol. This style of leader line was chosen as opposed to orthogonal leaders (i.e., leaders comprising one horizontal and one vertical segment). While in general, orthogonal leaders have been observed to be particularly legible [5], we have found that diagonal lines stand out better amidst the clutter of other marks in an equation (M6).

\subsection{Priming Glossary}

Scientific texts like textbooks often contain glossaries that allow readers to look up definitions of terms in a predictable place. One type of glossary that can be particularly helpful to readers is what Widdowson [102, page 82] called a "priming glossary," or a glossary that is shown to readers before a text to help prepare them for problematic words that may appear in the text. ScholarPhi prepends a priming glossary to scientific papers. The glossary includes a list of key terms and symbols, ordered by their appearance in the paper.

The glossary is intended to help readers in two ways. First, it lets them familiarize themselves with the nonce words that will be used in the paper. And second, it provides a reference that can be printed and viewed side-by-side with the paper. One advantage to presenting definitions in a priming glossary as opposed to tooltips is that definitions for all nonce words can be consolidated into one place (M3), allowing a reader to learn about groups of related nonce words all at once. Furthermore, the gloss provides scent (M4) indicating the density of nonce words, and the presence of definitions of those words, before the reader starts reading.

\section{IMPLEMENTATION}

For a given PDF document, the ScholarPhi interface requires information about the positions and definitions of the terms, symbols, and sub-symbols within it. This section briefly describes reference algorithms for obtaining this information. ${ }^{3}$ The algorithms analyze $\mathrm{T}_{\mathrm{E}} \mathrm{X} / \mathrm{ET} \mathrm{T} \mathrm{X}$-based PDFs to find exact locations of equations, symbols,

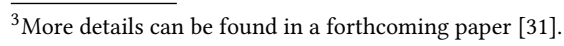

and sentences (Section 5.2), build up representations of composite symbols (Section 5.2.1), and detect definitions for symbols and terms (Section 5.3). This section also describes the implementation of the web-browser-based user interface (Section 5.4).

Because most scientific research today is published in PDFs (Portable Document Files), the ScholarPhi implementation tackles the challenging problem of providing interactions on PDFs. It would have been easier to demonstrate the technology on HTML or XML format, but that would not have achieved our goal of widespread use. For the same reason, we determined that it was important to provide the user interface for the document reader directly within a web browser without requiring a separate tool to be downloaded.

Algorithms for processing papers are implemented in $10.2 \mathrm{k}$ lines of Python code and 200 lines of custom $\mathrm{T}_{\mathrm{E}} \mathrm{X}$ coloring macros. The user interface is implemented in $10.5 \mathrm{k}$ lines of React code.

\subsection{Domain of Input Documents}

The algorithms below assume that a PDF has been compiled from a manuscript written in the $\mathrm{T}_{\mathrm{E}} \mathrm{X}$ or $\mathrm{LT}_{\mathrm{E}} \mathrm{X}$ typesetting language (collectively referred to as "TeX" below). It also assumes the sources for the manuscript are publicly available. This assumption holds for a broad collection of papers in computer science, where sources for papers are increasingly hosted on preprint servers like arXiv. In fact, arXiv hosts sources for over $1 \mathrm{M}$ papers [60, Section 2.2].

The algorithms operate on TeX rather than compiled PDF representations to improve the precision of detection of inline equations, the segmentation of equations into symbols, and the determination of which symbols are children of others. With TeX, these tasks become text parsing problems with existing, reliable solutions. The dependence on TeX is a stopgap; we anticipate that future implementations will accomplish these tasks by processing PDFs (e.g., [57]) or images (e.g., [75]) of papers directly.

\subsection{Nonce Word Position Detection}

Finding bounding boxes. To support definition tooltips, equation diagrams, and subsymbol selection, ScholarPhi requires bounding boxes for terms, equations, and symbols. To find these bounding boxes, our basic approach is to:

(1) Modify the TeX for a paper to assign a unique color to each term, equation, or symbol;

(2) Compile the modified TeX into a PDF;

(3) Render the PDF into images;

(4) Analyze the images to detect the colors assigned to each term, equation, or symbol.

This approach has proven successful in prior work for assembling datasets of bounding boxes for figures [91] and equations [56].

For example, consider the process of finding the bounding box for a single equation (Figure 4). First, equations are detected in the TeX sources for a paper with regular expressions that match equation environments (e.g., pairs of "\$” characters). Each equation is assigned a color by wrapping it with a TeX color command like " $\{\backslash$ color orange $\} ..$.$\} ". When the TeX is compiled, each equation$ appears in its assigned color.

The position of each equation is found by differencing the colorized PDF with the original PDF and finding a set of minimal bounding boxes that contain the assigned color. Boxes are found 


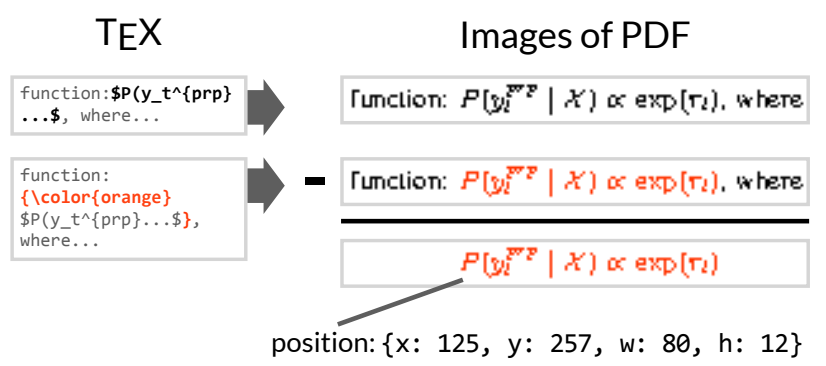

Figure 4: Image processing to find bounding boxes of equations, terms and symbols. Shown is a simple example of our approach. An equation is detected in a paper's TeX using text matching rules. The equation is colored using a TeX command. Then, the position of the equation is found by differencing the original PDF and the colorized PDF and detecting the colored pixels.

with a custom blob detector that eagerly creates boxes surrounding pixels of the same color in the same row of pixels, and then joins boxes appearing in adjacent rows. This blob detector detects the symbol " $y$ " as one bounding box, " $i$ " as two bounding boxes (with one box for the stem and one for the dot of the " $i$ "), and a multi-line equation as at least one box per line.

The strength of this approach over purely image-based or PDFbased recognition techniques lies in its ability to find bounding boxes of composite symbols, as described below. One limitation of this approach is that it requires TeX sources to be compiled many times for dense papers. Assuming a paper contains $N$ nonce words and an image processing library can distinguish between $C$ different colors, the TeX sources must be compiled at least $N$ / $C$ times to detect the positions of all nonce words.

5.2.1 Detecting Composite Symbols. The problem of segmenting TeX equations into symbols is already partially solved in open source tools like KaTeX [43] and MathJax [65], which convert TeX equations into structured MathML [27] documents. In these documents, nodes often correspond to symbols. For example, the TeX equation "x_i" would be parsed into the MathML document:

$$
<\text { msub }><\mathrm{mi}>\mathbf{x}</ \mathrm{mi}\rangle\langle\mathrm{mi}>\mathbf{i}</ \mathrm{mi}\rangle\langle/ \mathrm{msub}\rangle
$$

In this document, $<\mathrm{mi}>\mathbf{x}</ \mathrm{mi}>$ and $<\mathrm{mi}>\mathbf{i}</$ mi $>$ represent simple symbols, marked as mi or "identifier" elements. The document as a whole is one composite symbol, consisting of a subscripted symbol with $<\mathrm{mi}>\mathbf{x}</ \mathrm{mi}>$ as the base and $<\mathrm{mi}>\mathbf{i}</ \mathrm{mi}>$ as the script.

The KaTeX parser was extended to segment equations. The parser was instrumented to produce MathML documents where nodes are annotated with the positions of the characters they represent in the TeX. Then, the MathML document is searched for simple and composite symbols. Simple symbols are detected as identifier nodes, or rows of identifier nodes that can be merged into one word. Composite symbols of three types are detected:

- Scripts: subscripts, superscripts, or both. Detected as msub, msup, and msubsup nodes.

- Accents: hats, arrows, bars, etc. Detected as mover nodes with one operator (mo) child, and one identifier child.

\begin{tabular}{|c|c|c|c|c|}
\hline & script & accent & function & multiple \\
\hline TEX & $x \_i$ & $\backslash \operatorname{bar}\{x\}$ & $f(x)$ & $\backslash$ hat $\{\mathrm{p}\}\left(\mathrm{y}_{-} \mathrm{i} \backslash \mid \mathrm{x}\right)$ \\
\hline tokens & $x, i$ & $x$ & $f,(, x)$, & $p, y,(, i, x)$, \\
\hline $\begin{array}{r}\text { token } \\
\text { positions }\end{array}$ & $x_{i}$ & $\bar{x}$ & & $\hat{p}\left(y_{i} \mid x\right)$ \\
\hline affixes & - & $\backslash \operatorname{bar}\{x\}$ & - & $\backslash$ hat $\{p\}$ \\
\hline $\begin{array}{r}\text { affixed symbol } \\
\text { positions }\end{array}$ & - & $\bar{x}$ & - & $\hat{p}\left(y_{i} \mid x\right)$ \\
\hline $\begin{array}{c}\text { multi-token } \\
\text { symbols }\end{array}$ & $x \_i$ & - & $f(x)$ & $\underset{y_{-} i}{\backslash h a t}\{p\}\left(y_{-} i \backslash \mid x\right)$, \\
\hline $\begin{array}{r}\text { multi-token } \\
\text { ymbol positions }\end{array}$ & $x_{i}$ & - & $f(x)$ & $\hat{p}\left(y_{i} \mid x\right)$ \\
\hline
\end{tabular}

Figure 5: Detection of composite symbols. Symbols are segmented into tokens (i.e., individual characters). The positions of these tokens are found, and combined to find the bounding boxes of composite symbols.

- Functions: both declarations (e.g., $p(y \mid x))$ and usages (e.g., $f(2))$. Detected as an identifier followed by an opening parenthesis, a variable number of nodes, and a closing parenthesis.

The positions of simple symbols are detected using the TeX colorization technique described above. The positions of composite symbols are computed as the minimum bounding box that encapsulates all bounding boxes of simple symbols the composite symbol is made up of (Figure 5 shows some examples).

On a development set of 12 recent papers from recent proceedings of the ACL, EMNLP, NeurIPS, and ICML conferences, this technique identifies symbols (including both simple and composite symbols) with an average precision of $96 \%$ and recall of $88 \%$. Recall increases to $91 \%$ if TeX macros are expanded before processing. For the paper used as a stimulus in the usability study, this technique locates symbols with a precision of $98 \%$ and a recall of $98 \%$ (albeit omitting symbols that appeared in figures).

\subsection{Definition and Usage Recognition}

Definition Recognition. To recognize definitions of nonce words, our implementation has taken three approaches. The first approach is to leverage state-of-the-art natural language processing models for definition recognition. In research parallel to this project, we have developed new models for definition recognition [42], attaining state-of-the-art results with $73 \%$ precision and $74 \%$ recall on the W00 [41] dataset. We are continuously improving these models.

A second approach has been to identify abbreviations and expansions with state-of-the-art models, like those reviewed in Veyseh et al. [99]. These models regularly expand abbreviations with an accuracy above $90 \%$. A third approach appropriate for prototyping is to develop linguistic rules for extracting definitions, like searching for noun phrases that appear just before symbols, like the word "encoder" in the passage "The encoder $E$ is used to...". 
All of the above methods yield occasional errors. Section 7.3 envisions techniques for incorporating human input to improve the quality of definitions. As the above methods were fine-tuned on incompatible datasets without examples of nonce words or TeX symbols, they naturally do not detect them well (in our initial tests on the stimulus paper, abbreviation expansion with the Schwartz-Hearst algorithm [88] yields precision $=55 \%$, recall $=43 \%$; term $/$ symbol definition recognition with HEDDEx [42] yields precision $=24 \%$, recall $=6 \%$ ). To address this gap, we are developing datasets exclusively composed of nonce words to train more advanced models.

Identifying usages and defining formulae. To identify the usages of a nonce word, ScholarPhi extracts sentences from papers and associates each nonce word with the sentences it appears in. The pysbd [85] sentence boundary detector is applied to the TeX source for the paper; every sentence that the nonce word appears within is considered a usage. The positions of sentences within the PDF are found via the same colorization technique used to detect the positions of equations and symbols.

Defining formulae are extracted for symbols by searching for equations in which the symbol appeared on the left-hand side of an equation (i.e., to the left of a definition operator like "=").

\subsection{User Interface}

The ScholarPhi user interface is implemented as an overlay atop the Mozilla Foundation's open source $p d f . j s$ PDF reader application [69].

5.4.1 Reflowable Definitions and Usages with Math Expressions. In the ScholarPhi interface, definitions and usages are reflowable; that is, their text can wrap. This allows widgets like tooltips and lists of usages to have a reduced footprint, because definitions and usages can be rendered with a narrower width than the original text.

Widgets need to render math expressions cleanly, because math appears in many definitions and usages. Therefore, definitions and usages are rendered from the TeX for a sentence, using the KaTeX browser-based formula rendering library to transform TeX equations into resizable, reflowable HTML text elements.

5.4.2 Declutter. A paper is decluttered by applying a semi-opaque SVG mask over every page, and then subtracting from that mask rectangular regions that correspond to all appearances of a nonce word, and the sentences they belong to.

5.4.3 Symbol Search. When a user selects a symbol, the default search bar for "Control+F" text search is replaced with a navigation widget that lets users cycle through all appearances of the symbol When the user clicks out of the symbol, the default search widget is restored. Two symbols are considered to be the same symbol if they were parsed into the same MathML representation by our paper processing pipeline (see Section 5.2.1). Two symbols that are semantically the same may have different surface representations in the TeX (e.g., " $\{x\}$ ” versus “ $x$ ”). These surface differences typically disappear when the TeX is parsed into MathML.

5.4.4 Equation Diagrams. Equation diagrams are implemented as interactive labels and leader lines overlaid on the paper. Labels are shown for each symbol and sub-symbol for which a definition is available. If a symbol appears in the same equation diagram twice, only one instance of that symbol is labeled. Labels are placed on the top and bottom boundaries of an equation with a fixed margin between the edges of the equation and the labels. They are divided evenly between the top and bottom of the equation, with a preference to assign a label to the side of the equation (i.e., top or bottom) where it will be closest to the symbol it defines. Labels are spaced horizontally using Labella.js [53], which implements an overlap-free spacing algorithm introduced by Dwyer et al. [23].

\section{USABILITY STUDY}

We performed a formal remote usability study to ascertain the answers to the following questions: Do the features of ScholarPhi aid readers' ability to understand the use of nonce words when reading complex scientific papers? Do readers elect to use the features when given unstructured reading time? How are the features used to support the reading experience?

In a within-participants design, we compared the full features of ScholarPhi to a simplified version of the interface and a standard PDF reader on a series of close reading tasks on a machine learning paper. The quantitative and subjective results were strongly in favor of the affordances supplied by ScholarPhi over a standard PDF reader, with one exception.

\subsection{Study Design}

Participants. The criterion for inclusion was having previously read a machine learning paper. A total of 27 participants were recruited through university and company mailing lists. 18 were doctoral students, 5 were Master's students, 3 were undergraduate students, and 1 was a professional researcher. 13 of the 27 participants identified their discipline as machine learning, and 21 were somewhat or very comfortable with reading machine learning papers. Participants were compensated with a \$20 (USD) gift certificate. All study sessions were 1 hour long and held remotely over Zoom, a video conferencing platform; participant interactions were logged and screen activity was captured. Participants opened the application in a private browser window, and were asked to share their screen with the experimenters.

Stimulus paper. For this study, all participants read Linguisticallyinformed self-attention for semantic role labeling (LISA) [93]. (Several examples in Section 4 are drawn from this paper.) This paper was chosen as it is widely-read within the field of natural language processing, yet like many other papers, it uses some notation inconsistently and does not define all of its symbols explicitly.

As our goal was to assess interaction design independently of the performance of the algorithms (which are constantly evolving), a clean set of symbol, definition, and usage data was curated. Definitions were extracted by hand. Symbols, defining formulae, and usages were extracted automatically (Sections 5.2 and 5.3), with a small number of manual corrections. We are planning follow-up studies to examine the impact of errors on usability.

Tasks. Each session ran as follows: (1) Greeting and consent form. (2) Interactive tutorial with all features on a two-page paper [17]. (3) Read the abstract of the stimulus paper. (4) Complete a timed practice question with the full interface. (5) Complete three timed test questions using each of the three test interfaces (4 minutes each), each followed with a question about confidence and ease of 
use. (6) Unstructured reading of the stimulus paper (15-20 minutes).

(7) Questionnaire on background and subjective responses.

In the unstructured reading portion participants were encouraged to make use of the tools if they anticipated they would be helpful. The intention of this segment was to observe which aspects of the tool were used when not under time pressure.

Interfaces. Three interfaces were compared within-participants:

- "BASIC" is a basic PDF reader with standard search functionality (specifically, being able to find words using "Control-F" with a toggle button to match case and the ability to highlight all matches).

- "Declutter" is a PDF reader with additional declutter functionality.

- "ScholarPhi" is a PDF reader with all ScholarPhi features.

Test questions. The three multiple-choice test questions were each intended to assess a different aspect of pain points identified by formative studies.

- "Results": "According to Table 1, which model achieves the best recall on WSJ data when GloVE embeddings are used?"

- "DATASET": "Which text corpora is the ConLL-2005 dataset made from? Select all that apply."

- "Symbols": "What does T (upper case) mean in this paper? Select all senses in which T is used."

Assessment measures. For each of the test questions, we measured the following quantitative metrics:

- "CONFIDENCE" is a five-point Likert scale variable indicating the participant's self-assessment of the following prompt: "I am confident I came up with the right answer." A score of 5 indicates strong agreement, and a score of 1 indicates strong disagreement.

- "EASE" is a five-point Likert scale variable indicating the participant's self-assessment of the following prompt: "It was easy to find the answer." A score of 5 indicates strong agreement, and a score of 1 indicates strong disagreement.

- "TIME" is the number of seconds the participant spent to answer the question. It is measured from when the question first appeared on the participant's screen, to when the participant clicked the next button or the question timer expired (whichever event occurred first).

- "Correct" is a binary variable indicating whether the participant's response was correct. For questions requiring a response with multiple selections, a response was considered correct if it included all and only the correct selections.

- "AREA" is the proportion of the full paper viewed. It is computed as the cumulative total area viewed over the total area in the entire paper. It ranges between values 0 (none of the paper viewed) and 1 (entire paper viewed).

- "Distance" is a continuous variable measuring the cumulative (normalized) absolute vertical pixel distance-that is, number of document lengths-traversed by a participant. Normalization controls for different pixel heights across participants' devices. The distance between the top and bottom pixels on each page is set to $1 / n_{\text {pages }}$ such that the entire paper's total height sums to 1.0; traversing the length of the paper twice would contribute 2.0 to the total DisTANCE.

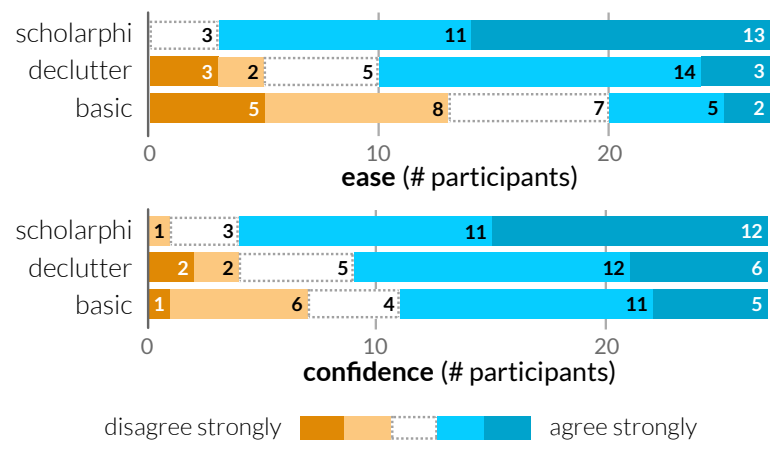

Figure 6: Subjective responses for test questions. Larger counts of agreement are preferred. Both measures were reported on an ordinal scale with levels "disagree strongly," "disagree somewhat," "neutral," "agree somewhat," and "agree strongly."

Unstructured reading task measurements. Measurements in the unstructured reading tasks included frequency of usage of key features and subjective feedback.

Assignment. Using a repeated measures factorial design, we assigned each participant to three of nine possible configurationsinterface-question pairs-while ensuring that (i) each participant observed each interface and each question type exactly once and (ii) all nine configurations had the same number of assigned participants. Assignment was counterbalanced such that no one interface, question, or interface-question pair was experienced more often than others as the first, second, or third task. 9 participants received interfaces in the order [Basic (B), Declutter (D), ScholarPhi (S)], 9 in the order [D, S, B], and 9 in the order [S, B, D].

Analysis. For each of the quantitative measurements, we fit a generalized linear mixed-effects model (GLMM) with fixed effects for the interface and question factors (and a fixed-effects interaction term). Details can be found in Appendix A.1.

Reduced controls due to remote testing. Since the study was held remotely, some standard controls could not be employed: the size of the screen, the speed of the user's computer (the PDF reader appeared to have lag for some participants and not for others), and the distraction in the environment (background noise could be heard for many of the participants). These differences might account for variation in performance and subjective accounts of the experience. Rather than degrading the quality of the data, these factors make the study better represent the variation that we anticipate readers using this tool would have in their environments.

\subsection{Quantitative Results}

Figures 6, 7, and 8 summarize how the measures on the test questions vary across the three interfaces. We report results from twosided tests for pairwise differences in mean effects between interfaces in Table 2. These results indicate which trends in the figures are statistically significant at the $\alpha=0.05$ level.

In terms of the subjective scores, we observed that ScHOLARPHI outperformed BASIC on EASE and CONFIDENCE, and Declutter on Ease. Declutter also reported higher Ease than Basic, but 


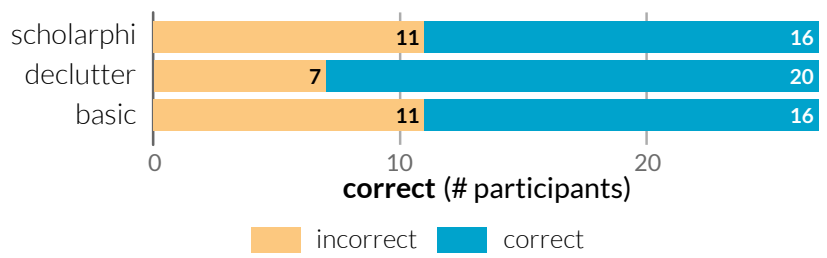

Figure 7: Correctness of responses for test questions. A lower number of incorrect answers is preferred.

not higher CONFIDENCE. SCHOLARPhi reported higher Confidence than Declutter, but this result was not significant at $\alpha=0.05$.

ScholarPHi outperformed the other interfaces in terms of time required to answer the test questions (TIME) (DECLUTTER and BASIC were not significantly different). No statistically significant differences were observed in the number of participants who answered questions correctly among the three interfaces.

Finally, we observed that participants traversed less screen DisTANCE and viewed less AREA of the paper under ScholarPhi and Declutter compared to BAsic; ScholarPhi outperformed DeCLUTTER on AREa but did not significantly outperform DeclutTer on Distance. Overall, these results suggest that even the lighterweight version of the tool, with the declutter overlay alone, yields benefits over the standard PDF reader, but the full set of features in SCHOLARPHI is especially beneficial.

Upon further inspection of the results on CoRrect, we found the performance of participants on a particular question yielded the reason for SCHOLARPHI performing similarly to BASIC (with DECLUTTER yielding slightly higher results): participants performed better on both the Results and Dataset questions using SchOLARPHI, but performed very poorly on the Symbols question with this interface. Recall from Section 3.3 (M1) that the stimulus paper uses the symbol $T$ inconsistently and also does not define all senses of this symbol. We found that participants almost always answered this question incorrectly using SCHOLARPHI because the definitions list did not show all of the usages, and the participants had the expectation that the definitions list showed all senses of the symbol. This highlights an important potential drawback of a tool like ScholarPhi: it can mislead if it implies incorrect information.

\subsection{Qualitative Results}

When describing qualitative results, we refer to participants as "readers," and to individual readers with pseudonyms P1-27.

6.3.1 Subjective Impressions. Subjective responses were obtained both from oral comments during the study and from open-ended questions in the final questionnaire. Readers' impressions of ScholarPhi were overwhelmingly positive. Readers were enthusiastic about the support that ScholarPhi provided for the reading task. They described the tool as "cool" (P8), "very cool", (P13), "super cool" (P12), and "amazing" (P4, P16, P19). Eight of the 27 responses to the open-ended questionnaire forms contained exclamation marks conveying reader excitement for the tool. Several readers commented on the polish of the prototype (P7, P24).

Readers described three supporting roles they envisioned ScholarPhi playing during reading tasks. First, they believed ScholarPhi

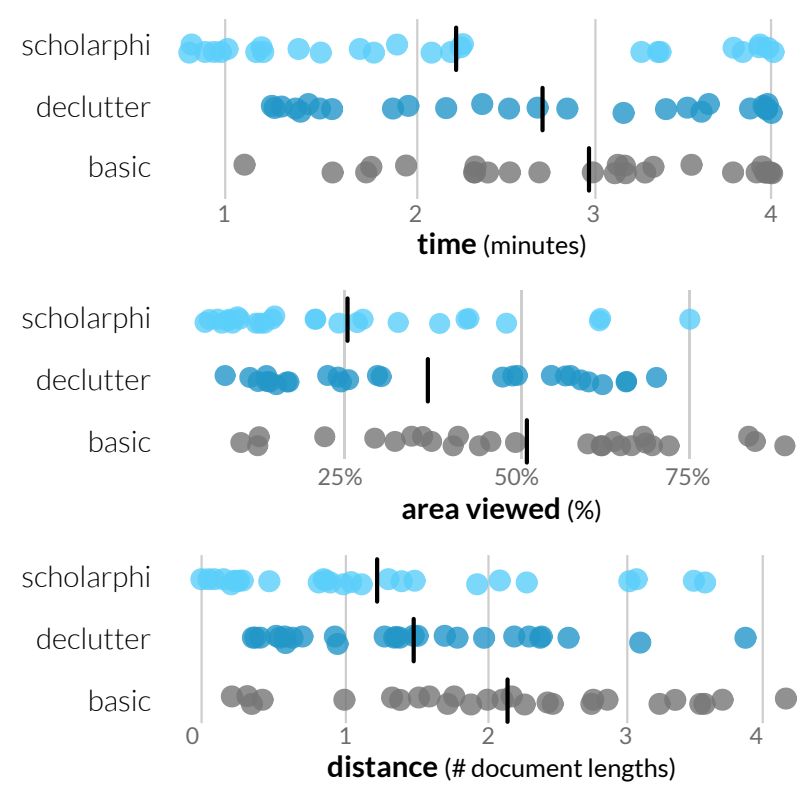

Figure 8: Quantitative results for test questions. The vertical bars indicate the mean. Lower values are preferred.

would help them maintain "reading flow" (P16, P27). In the words of one reader, ScholarPhi helped them "focus on the aspects of the paper that interested me, and not waste time on other stuff" like reminding themselves of definitions (P4). The features provided timely reminders (P10, P21, P26), and eliminated the need to traverse "back and forth" within the paper (P11).

Second, ScholarPhi helped them "check their understanding" of the meanings of nonce words (P16) and the passages of text they appeared in (P20). Third, readers believed ScholarPhi could help them understand papers that they otherwise would not have had the vocabulary to read easily (P4, P23), in effect "lowering the barrier" to reading papers in fields outside of one's expertise.

Anticipated usage. To determine which of ScholarPhi's features would be of greatest interest to readers in the future, and hence which features should be developed further, readers were asked to report how often they expected they would use each feature if it was available in the software they used to read papers. Expected frequency was reported on a five-point ordinal scale ("Never," "Rarely," "Sometimes," "Often," "Always," and "Unsure").

Readers expected they would use most features often. They envisioned using multiple features very frequently, including definition tooltips for symbols (16 "always"; 8 "often"), definition tooltips for terms $(15 ; 9)$, and equation diagrams $(17 ; 6)$. The features of decluttering for symbols $(5 ; 13)$ and terms $(2 ; 15)$, and the priming glossary $(8 ; 6)$, were envisioned as being used less frequently. While a reader could indicate they "never" saw themselves using a feature, not a single reader selected this option for any feature.

6.3.2 Use of Features. To identify strengths in the design and opportunities for improvement, usage logs were inspected, and participant feedback on individual features was reviewed. All readers 


\begin{tabular}{rcccccc}
\hline & $\hat{y}_{S}-\hat{y}_{D}$ & $p$ & $\hat{y}_{D}-\hat{y}_{B}$ & $p$ & $\hat{y}_{S}-\hat{y}_{B}$ & $p$ \\
\hline CONFIDENCE $(1-5)$ & 0.59 & 0.094 & 0.19 & 0.785 & 0.78 & $\mathbf{0 . 0 2 0}$ \\
EASE (1-5) & 0.93 & $\mathbf{0 . 0 0 5}$ & 0.78 & $\mathbf{0 . 0 2 0}$ & 1.70 & $<\mathbf{0 . 0 0 0 1}$ \\
TIME (seconds) & -27.6 & $\mathbf{0 . 0 1 5}$ & -16.8 & 0.218 & -45.4 & $\mathbf{0 . 0 0 0 1}$ \\
CORRECT & $-15 \%$ & 0.393 & $15 \%$ & 0.393 & $0 \%$ & 1.000 \\
Distance (\# doc lengths) & -0.24 & 0.572 & -0.66 & $\mathbf{0 . 0 2 3}$ & -0.90 & $\mathbf{0 . 0 0 1}$ \\
AREA & $-11 \%$ & $\mathbf{0 . 0 4 7}$ & $-14 \%$ & $\mathbf{0 . 0 0 9}$ & $-25 \%$ & $<\mathbf{0 . 0 0 0 1}$ \\
\hline
\end{tabular}

Table 2: Two-sided tests for pairwise differences in mean effects between interfaces. This table reports $\hat{y}_{i}-\hat{y}_{j}$ and Holm-Bonferronicorrected $p$-values [36], where $\hat{y}$ is the estimated mean of $y$ under the GLMM, and $i, j$ correspond to interface options $-B=$ BAsIC, $D=$ Declutter, $S=$ ScholarPhi. For example, in the cell for (TIme, $\hat{y}_{S}-\hat{y}_{B}$ ), we can interpret the result as ScholarPhi is associated with tasks completed in 45.4 fewer seconds in TIME than BASIC, on average. CoRRECT and AREA differences are reported as absolute, not relative, percentage point differences. Statistically significant $p$-values are bolded. Further details about this analysis appear in Appendix A.1.

except for one (96\%) used at least one of ScholarPhi's features during the unstructured reading time. Analysis of the aforementioned data led to the following observations about feature design:

Definition tooltips. For most readers, tooltips were ScholarPhi's most essential feature. During unstructured reading time, readers used definition tooltips more than any other feature. All but three readers opened at least one tooltip for a symbol, and all but one reader opened at least one tooltip for a term. When readers used tooltips they used them often. Readers opened tooltips for symbols a median of 10 times $(\sigma=13.8, \max =54)$, and for terms a median of 5 times $(\sigma=3.6, \max =14)$.

Tooltips served two purposes for readers. The first was the purpose they were designed for: to provide access to definitions of nonce words that appeared elsewhere in the paper (P10). A second purpose was to help a reader check whether the passage the reader was consulting was indeed the definition of a nonce word, which could help a reader make sure they were not missing other information of interest about the nonce word (P2).

Declutter. In contrast to tooltips, which were unanimously appreciated, the declutter feature saw disagreement. Some readers valued the feature, and others did not.

On the whole, readers' behaviors suggests that most readers expected declutter to be useful for finding answers to questions in a paper: all participants activated declutter at least once in the test task where they used an interface with only the declutter feature enabled. Several readers explicitly told us they believed declutter could be useful for finding information about nonce words (P6, P11, P15, P23, P26). Readers reported that the feature made the paper look "less cluttered," and that it could help them feel "less overwhelmed" by the text in the paper (P27).

Other readers indicated gaps in the design. Some readers did not understand the point of the feature (P25), or thought it provided little value over the definition tooltips (P22). Others felt that the standard "Control-F" search provided a more efficient interface for searching a paper than scrolling through a paper with declutter (P2). An additional gap of the feature is that, unlike "Control-F" search, declutter cannot be invoked unless the nonce word of interest is already in view. One reader believed this would be frustrating in the scenario where they temporarily deactivated declutter in order to read the low-lighted text and then wished to resume declutter for the same nonce word as before (P14).

Lists of usages. Nearly all (20 of 27) readers opened a list of definitions, defining formulae, or usages during the unstructured reading task. 18 readers opened a list of definitions, 3 opened a list of defining formulae, and 10 opened a list of usages. Some readers used the lists heavily. For instance, one participant opened the lists of definitions and usages eight times each (P4).

Readers reported that they used the list of usages to develop an understanding of the purpose of the paper (P9) and gather context to check their understanding of a term (P16). One reader described the list of usages as a "guide" to support non-linear reading (P27). They navigated the paper by iteratively selecting nonce words, reviewing usages, jumping to a usage, and then looking for other nonce words of interest in the passage they jumped to. This reader believed the list helped them answer questions as they came up, rather than waiting them to be resolved in a later passage.

Equation diagrams. More readers expected they would "always" use equation diagrams for future readings than any other feature. Almost all (21 of 27) readers opened an equation diagram during the unstructured reading task. Most readers opened multiple, with the median reader opening $3(\sigma=4.3, \max =14)$.

The primary use of equation diagrams was to understand the symbols in an equation without attending to the surrounding text (P1, P6, P11, P13, P14, P21, P24). Diagrams were seen as particularly useful when an equation was long (P24) or complex (P11). One of the equations, for instance, consisted of four lines of notation with a total of fourteen symbols for which definitions were available, and many others for which definitions were not. Readers were regularly observed pausing to study this equation with the diagram open.

Beyond the primary use of describing symbols, one reader described diagrams as supporting a new way of navigating the text. This reader skimmed the technical section of the paper by opening the diagrams one-by-one, familiarizing themselves with the section by reading the equations rather than the prose (P7).

Priming glossary. The priming glossary was the least-used feature during the unstructured reading task. A few readers (6 of 27) were observed consulting the priming glossary for a nontrivial amount of time, defined in our protocol to be 10 or more seconds. 
Although readers infrequently consulted the priming glossary, a few readers believed it would be useful under certain circumstances. Some readers believed the glossary could help them orient to the terminology used in a paper before reading it (P13, P16). In line with this claim, one reader spent 2 minutes (P16) and another spent 5 minutes (P1) carefully studying the glossary at the beginning of the unstructured reading time. Second, readers indicated an expectation that the glossary would provide more thorough definitions of nonce words than the tooltips. Several readers appeared to visit the glossary as a fallback when the definition tooltip did not contain the information they sought (P3, P12, P14, P22).

Use of features in concert. While ScholarPhi's features were often used in isolation, we also observed on several equations readers using several disparate features in rapid succession. For example, P6 clicked an equation to reveal a diagram, selected one of the symbols in the diagram, opened the list of definitions for the symbol, and then clicked on a link that took them to one of those definitions. Several readers chained interactions across multiple of ScholarPhi's features in a similar way (P6, P8, P13, P19).

\section{DISCUSSION AND FUTURE WORK}

\subsection{Summary of Results}

The outcomes of the usability study produced the following answers to the research questions:

Do the features of ScholarPhi aid readers' ability to understand the use of nonce words when reading complex scientific papers? Yes. When asked to answer questions requiring understanding of nonce words, readers answered questions significantly more quickly with ScholarPhi than with a baseline PDF reader, while viewing significantly less of the paper.

Do readers elect to use the features when given unstructured reading time? Yes. $96 \%$ of readers used ScholarPhi's features at least once during 15 minutes of unstructured reading time. Tooltips were the most frequently used feature: readers opened a median of 10 tooltips for symbols, and 5 for terms. Equation diagrams were opened a median of 3 times. Almost all participants opened a list of definitions, defining formulae, or usages at least once.

How are the features used to support the reading experience? On the whole, readers used the features for the reasons expected: they referred to tooltips to remind themselves of forgotten definitions, activated declutter to find information about nonce words within a less cluttered view of the paper, and opened equation diagrams to view the definitions of many symbols at once. Readers also used the tools to support the reading experience in unconventional ways, for instance using the list of usages as a "guide" to support a non-linear, curiosity-driven reading, and skimming a section by jumping from one equation diagram to the next.

\subsection{Limitations}

A major limitation of the usability study is its focus on a single paper, where performance was measured for only three tasks. Papers vary widely in clarity and readability. To improve generalizability of the study, the paper was selected to be a widely-read scientific paper exhibiting some of the very problems the system was seeking to address. Furthermore, the three tasks were chosen to require an understanding of different types of nonce words: terms referring to datasets, baselines, and symbols. In the future, we will continue to evaluate ScholarPhi on a variety of research papers, as has been done to date through the iterative design process for the tool.

A second limitation, that pertains to the tool's suitability for supporting unstructured reading, is that readers in the study only used the tool for 15-20 minutes, and may have not had enough time to discover limitations that would preclude them using the tool in the future. Observations from our pilot studies have suggested that readers continue to find aspects of the tool useful after 20 minutes of reading, but longitudinal studies are necessary to better assess how readers would employ ScholarPhi in day-to-day use.

\subsection{Future Work}

The study of ScholarPhi has revealed three opportunities for future research to advance the potential of intelligent reading interfaces to aid in the authoring and reading of scientific papers.

Connecting Readers to Definitions Beyond the Paper. The larger vision of ScholarPhi is to help scientists more easily read papers by linking relevant information to its location of use. This includes providing links to the contents of cited papers, and providing definitions going beyond nonce words to terms defined externally to the paper. Indeed, readers in the formative study, pilot studies, and usability study all asked for the ability to look for definitions of terms that resided outside of a paper. Future work will incorporate this information into the ScholarPhi reader.

Co-development of Reading Interfaces and Machine Learning Models. Machine learning models are imperfect; our own recent research [42] shows that the state-of-the-art algorithms for definition detection currently have a problem of recall when it comes to detecting definitions in scientific papers. Researchers in human-computer interaction have explored how users interact with imperfect AI algorithms [48, 105]. ScholarPhi may benefit from an analogous thread of research which explores how models for augmenting texts with interactive affordances can convey uncertainty.

Definition quality could also be improved by incorporating human input. Annotation tools could let authors explicitly define nonce words and then refer to them unambiguously. Furthermore, readers could be asked to improve definitions by selecting helpful definitions from among a set of alternatives, or directly editing the definitions shown in tooltips and equation diagrams.

ScholarPhi for Writing Scientific Papers. A dual of ScholarPhi could support the task of writing clear scientific papers. Such a tool could indicate to an author when they left a nonce word undefined, when they used the same symbol to mean two different things (as is often the case for symbols like " $k$ "), and to know when they are using multiple nonce words to refer to the same idea. The same paper processing technologies that can detect definitions and relate two nonce words to each other could suit writing just as well as reading. As we saw in the development of ScholarPhi, the design exploration of augmented writing interfaces likely needs to begin with careful observations of writers to understand how lightweight, non-intrusive features can support the writing task without distracting authors. 


\section{CONCLUSION}

The ScholarPhi system was designed to help readers concentrate on the cognitively demanding task of reading scientific papers by providing them efficient access to definitions of nonce words. The iterative design of the system revealed that systems like ScholarPhi need to tailor definitions to the passage where a reader seeks an understanding of a nonce word, provide scent, and avoid distracting readers from their reading. A usability study with 27 researchers showed that when using ScholarPhi versus a standard PDF reader, they could answer questions that required an understanding of nonce words in less time, viewing less of the paper. Readers could see using ScholarPhi's definition tooltips and equation diagrams "often" or "always" if they were available in their reading interface. These strong empirical results suggest that researchers are eager and ready for tools like ScholarPhi that support the reading task by providing just-in-time, position-sensitive definitions of nonce words when and where they need them.

\section{ACKNOWLEDGMENTS}

Zachary Kirby, Jocelyn Sun, Luming Chen, Nidhi Kakulawaram, RJ Pimentel, and Benjamin Barantschik contributed to the design, implementation, and evaluation of prototypes of ScholarPhi. Luca Weihs, Brendan Roof, and Alvaro Herrasti developed a prototype algorithm for locating equations in $\mathrm{AT}_{\mathrm{E}} \mathrm{X}$ papers which inspired the approach used to locate symbols and terms in this paper. Luca Weihs and Amrit Dhar provided feedback on the statistical analysis. This work would not have been possible without their contributions.

This research receives funding from the Alfred P. Sloan Foundation, the Allen Institute for AI, Office of Naval Research grants N00014-15-1-2774 and N00014-18-1-2193, National Science Foundation RAPID grant 2040196, and the Washington Research Foundation Thomas J. Cable Professorship.

\section{REFERENCES}

[1] Takeshi Abekawa and Akiko Aizawa. 2016. SideNoter: Scholarly Paper Browsing System based on PDF Restructuring and Text Annotation. In Proceedings of the International Conference on Computational Linguistics. International Conference on Computational Linguistics, 136-140.

[2] Annette Adler, Anuj Gujar, Beverly L. Harrison, Kenton O'Hara, and Abigail Sellen. 1998. A diary study of work-related reading: design implications for digital reading devices. In Proceedings of the CHI Conference on Human Factors in Computing Systems. ACM, 241-248.

[3] Lara Alcock. 2009. e-Proofs: Student Experience of Online Resources to Aid Understanding of Mathematical Proofs. In Proceedings of the Conference on Research in Undergraduate Mathematics Education. Special Interest Group of the Mathematical Association of America on Research in Undergraduate Mathematics Education.

[4] Sriram Karthik Badam, Zhicheng Liu, and Niklas Elmqvist. 2019. Elastic Documents: Coupling Text and Tables through Contextual Visualizations for Enhanced Document Reading. IEEE Transactions on Visualization and Computer Graphics 25, 1 (January 2019), 661-671.

[5] Lukas Barth, Andreas Gemsa, Benjamin Niedermann, and Martin Nöllenburg. 2019. On the readability of leaders in boundary labeling. Information Visualization 18, 1 (2019), 110-132.

[6] Douglas Bates, Martin Mächler, Benjamin M. Bolker, and Steven C. Walker. 2015 Fitting Linear Mixed-Effects Models Using lme4. Fournal of Statistical Software 67, 1 (October 2015), 1-48.

[7] Charles Bazerman. 1985. Physicists Reading Physics: Schema-Laden Purposes and Purpose-Laden Schema. Written Communication 2, 1 (January 1985), 3-23.

[8] Eric A. Bier, Edward W. Ishak, and Ed Chi. 2006. Entity Quick Click: Rapid Text Copying Based on Automatic Entity Extraction. In Proceedings of the CHI Conference on Human Factors in Computing Systems. ACM, 562-567.

[9] Vannevar Bush. 1945. As we may think. The Atlantic 176, 1 (July 1945), 101-108.

10] Isabel Cachola, Kyle Lo, Arman Cohan, and Daniel Weld. 2020. TLDR: Extreme Summarization of Scientific Documents. In Findings of the Association for Computational Linguistics: EMNLP 2020. Association for Computational Linguistics,
4766-4777.

[11] Bay-Wei Chang, Jock D. Mackinlay, Polle T. Zellweger, and Takeo Igarashi. 1998. A Negotiation Architecture for Fluid Documents. In Proceedings of the Symposium on User Interface Software and Technology. ACM, 123-132.

[12] Joseph Chee Chang, Nathan Hahn, and Aniket Kittur. 2016. Supporting Mobile Sensemaking Through Intentionally Uncertain Highlighting. In Proceedings of the Symposium on User Interface Software and Technology. ACM, 61-68.

[13] Ying-Hsueh Cheng and Robert L. Good. 2009. L1 glosses: Effects on EFL learners' reading comprehension and vocabulary retention. Reading in a Foreign Language 2009, 2 (October 2009), 119-142.

[14] Parmit K. Chilana, Amy J. Ko, and Jacob O. Wobbrock. 2012. LemonAid: Selection-Based Crowdsourced Contextual Help for Web Applications. In Proceedings of the CHI Conference on Human Factors in Computing Systems. ACM, $1549-1558$.

[15] Rune Haubo B Christensen. 2018. Cumulative Link Models for Ordinal Regression with the R Package ordinal. (2018). http://cran.uni-muenster.de/web/ packages/ordinal/vignettes/clm_article.pdf.

[16] Avital Cnaan, Nan M. Laird, and Peter Slasor. 1997. Using the general linear mixed model to analyse unbalanced repeated measures and longitudinal data. Statistics in Medicine 16, 20 (1997), 2349-2380.

[17] Joseph Paul Cohen, Henry Z. Lo, Tingting Lu, and Wei Ding. 2016. Crater Detection via Convolutional Neural Networks. (2016). arXiv:1601.00978 [cs.CV]

[18] Jeff Conklin. 1987. Hypertext: An Introduction and Survey. Computer 20, 9 (September 1987), 17-41.

[19] Robert Cudeck. 1996. Mixed-effects Models in the Study of Individual Differences with Repeated Measures Data. Multivariate Behavioral Research 31, 3 (1996), 371-403.

[20] Isabelle De Ridder. 2002. Visible or invisible links? In Proceedings of the CHI Conference on Human Factors in Computing Systems. ACM, 624-625.

[21] Diana DeStefano and Jo-Anne LeFevre. 2007. Cognitive load in hypertext reading: A review. Computers in Human Behavior 23, 3 (May 2007), 1616-1641.

[22] Pierre Dragicevic, Stéphane Huot, and Fanny Chevalier. 2011. Gliimpse: Animating from Markup Code to Rendered Documents and Vice Versa. In Proceedings of the Symposium on User Interface Software and Technology. ACM, 257-262.

[23] Tim Dwyer, Kim Marriott, and Peter J. Stuckey. 2005. Fast Node Overlap Removal. In International Symposium on Graph Drawing. Springer, 153-164.

[24] Dennis E. Egan, Joel R. Remde, Louis M. Gomez, Thomas K. Landauer, Jennifer Eberhardt, and Carol C. Lochbaum. 1989. Formative Design-Evaluation of SuperBook. ACM Transactions on Information Systems 7, 1 (1989), 30-57.

[25] eLife. 2013. Seeing through the eLife Lens: A new way to view research. (2013). https://elifesciences.org/inside-elife/0414db99/seeing-through-the-elifelens-a-new-way-to-view-research.

[26] Fermat's Library. https://fermatslibrary.com/. Last accessed September 16, 2020.

[27] Max Froumentin. Mathematical Markup Language (MathML). https://www. w3.org/Math/whatIsMathML.html. Last accessed September 16, 2020.

[28] Jamey Graham. 1999. The Reader's Helper: A Personalized Document Reading Environment. In Proceedings of the CHI Conference on Human Factors in Computing Systems. ACM, 481-488.

[29] Tovi Grossman, Fanny Chevalier, and Rubaiat Habib Kazi. 2015. Your Paper is Dead! Bringing Life to Research Articles with Animated Figures. In Proceedings of the CHI Conference on Human Factors in Computing Systems. ACM, 461-475.

[30] Andrew Head, Codanda Appachu, Marti A. Hearst, and Björn Hartmann. 2015. Tutorons: Generating Context-Relevant, On-Demand Explanations and Demonstrations of Online Code. In Proceedings of the Symposium on Visual Languages and Human-Centric Computing. IEEE, 3-12.

[31] Andrew Head, Kyle Lo, Daniel S. Weld, and Marti A. Hearst. Forthcoming. Recognition of Composite Symbols, Their Components, and Their Locations in LaTeX-Based PDFs. (Forthcoming).

[32] Marti A. Hearst, Emily Pedersen, Lekha Patil, Elsie Lee, Paul Laskowski, and Steven Franconeri. 2020. An Evaluation of Semantically Grouped Word Cloud Designs. IEEE Transactions on Visualization and Computer Graphics 26, 9 (September 2020), 2748-2761.

[33] William C. Hill, James D. Hollan, Dave Wroblewski, and Tim McCandless. 1992. Edit wear and read wear. In Proceedings of the CHI Conference on Human Factors in Computing Systems. ACM, 3-9.

[34] Terje Hillesund. 2010. Digital reading spaces: How expert readers handle books, the Web and electronic paper. First Monday 15, 4 (April 2010).

[35] Ken Hinckley, Xiaojun Bi, Michel Pahud, and Bill Buxton. 2012. Informal Information Gathering Techniques for Active Reading. In Proceedings of the CHI Conference on Human Factors in Computing Systems. ACM, 1893-1896.

[36] Sture Holm. 1979. A Simple Sequentially Rejective Multiple Test Procedure. Scandinavian fournal of Statistics 6, 2 (1979), 65-70.

[37] Jessica Hullman, Yea-Seul Kim, Francis Nguyen, Lauren Speers, and Maneesh Agrawala. 2018. Improving Comprehension of Measurements Using Concrete Re-Expression Strategies. In Proceedings of the CHI Conference on Human Factors in Computing Systems. ACM. Paper 34. 
[38] Matthew Inglis and Lara Alcock. 2012. Expert and Novice Approaches to Reading Mathematical Proofs. Fournal for Research in Mathematics Education 43, 4 (July 2012), 358-390.

[39] Nan Jiang and Huseyin Dogan. 2014. CrowdHiLite: A Peer Review Service to Support Serious Reading on the Screen. In Proceedings of the International BCS Human Computer Interaction Conference. British Computer Society, 323-328.

[40] Zhuoren Jiang, Liangcai Gao, Ke Yuan, Zheng Gao, Zhi Tang, and Xiaozhong Liu. 2018. Mathematics Content Understanding for Cyberlearning via Formula Evolution Map. In Proceedings of the International Conference on Information and Knowledge Management. ACM, 37-46.

[41] Yiping Jin, Min-Yen Kan, Jun-Ping Ng, and Xiangnan He. 2013. Mining Scientific Terms and their Definitions: A Study of the ACL Anthology. In Proceedings of the Conference on Empirical Methods in Natural Language Processing. Association for Computational Linguistics, 780-790.

[42] Dongyeop Kang, Andrew Head, Risham Sidhu, Kyle Lo, Daniel Weld, and Marti A. Hearst. 2020. Document-Level Definition Detection in Scholarly Documents: Existing Models, Error Analyses, and Future Directions. In Proceedings of the First Workshop on Scholarly Document Processing. Association for Computational Linguistics, 196-206.

[43] KaTeX. https://katex.org. Last accessed September 16, 2020

[44] Caitlin Kelleher and Randy Pausch. 2005. Stencils-Based Tutorials: Design and Evaluation. In Proceedings of the CHI Conference on Human Factors in Computing Systems. ACM, 541-550.

[45] Dae Hyun Kim, Enamul Hoque, Juho Kim, and Maneesh Agrawala. 2018. Fa cilitating Document Reading by Linking Text and Tables. In Proceedings of the Symposium on User Interface Software and Technology. ACM, 423-434.

[46] Yea-Seul Kim, Jessica Hullman, Matthew Burgess, and Eytan Adar. 2016. Simple Science: Lexical Simplification of Scientific Terminology. In Proceedings of the Conference on Empirical Methods in Natural Language Processing. Association for Computational Linguistics, 1066-1071.

[47] Amy J. Ko and Brad A. Myers. 2009. Finding Causes of Program Output with the Java Whyline. In Proceedings of the CHI Conference on Human Factors in Computing Systems. ACM, 1569-1578.

[48] Rafal Kocielnik, Saleema Amershi, and Paul N. Bennett. 2019. Will You Accept an Imperfect AI? Exploring Designs for Adjusting End-user Expectations of AI Systems. In Proceedings of the CHI Conference on Human Factors in Computing Systems. ACM. Paper 411.

[49] Andrea Kohlhase, Michael Kohlhase, and Taweechai Ouypornkochagorn. 2018 Discourse Phenomena in Mathematical Documents. In Proceedings of the Con ference on Intelligent Computer Mathematics. Springer, 147-163.

[50] Michael Kohlhase, Joseph Corneli, Catalin David, Deyan Ginev, Constantin Jucovschi, Andrea Kohlhase, Christoph Lange, Bogdan Matican, Stefan Mirea and Vyacheslav Zholudev. 2011. The Planetary System: Web 3.0 \& Active Documents for STEM. Procedia Computer Science 4 (2011), 598-607.

[51] Nicholas Kong, Marti A. Hearst, and Maneesh Agrawala. 2014. Extracting References Between Text and Charts via Crowdsourcing. In Proceedings of the CHI Conference on Human Factors in Computing Systems. ACM, 31-40.

[52] Alexandra Kuznetsova, Peter Brockhoff, and Rune H. B. Christensen. 2017. lmerTest Package: Tests in Linear Mixed Effects Models. Journal of Statistical Software 82, 13 (December 2017), 1-26.

[53] Labella.js. https://twitter.github.io/labella.js/. Last accessed September 16, 2020.

[54] Elina Late, Carol Tenopir, Sanna Talja, and Lisa Christian. 2019. Reading prac tices in scholarly work: from articles and books to blogs. Fournal of Documentation 75, 3 (2019), 478-499.

[55] Juho Lee, Yoonho Lee, and Yee Whye Teh. 2019. Deep Amortized Clustering. (2019). arXiv:1909.13433 [cs.LG]

[56] Minghao Li, Yiheng Xu, Lei Cui, Shaohan Huang, Furu Wei, Zhoujun Li, and Ming Zhou. 2020. DocBank: A Benchmark Dataset for Document Layout Analysis. In Proceedings of the International Conference on Computational Linguistics. International Committee on Computational Linguistics, 949-960.

[57] Xiaoyan Lin, Liangcai Gao, Zhi Tang, Xiaofan Lin, and Xuan Hu. 2011. Mathematical Formula Identification in PDF Documents. In Proceedings of the International Conference on Document Analysis and Recognition. IEEE, 1419-1423.

[58] Mary J. Lindstrom and Douglas M. Bates. 1990. Nonlinear Mixed Effects Models for Repeated Measures Data. Biometrics 46, 3 (September 1990), 673-687.

[59] Xiaozhong Liu, Zhuoren Jiang, and Liangcai Gao. 2015. Scientific Information Understanding via Open Educational Resources (OER). In Proceedings of the International Conference on Research and Development in Information Retrieval. ACM, 645-654.

[60] Kyle Lo, Lucy Lu Wang, Mark Neumann, Rodney Kinney, and Daniel S. Weld. 2020. S2ORC: The Semantic Scholar Open Research Corpus. In Proceedings of the Annual Meeting of the Association for Computational Linguistics. Association for Computational Linguistics, 4969-4983.

[61] Look Up Words, People, and Places While You Read. https://www.amazon.com/ $\mathrm{b}$ ?ie=UTF8\&node=17717476011. Last accessed September 16, 2020.

[62] Mircea F. Lungu, Luc van den Brand, Dan Chirtoaca, and Martin Avagyan. 2018 As We May Study: Towards the Web as a Personalized Language Textbook. In
Proceedings of the CHI Conference on Human Factors in Computing Systems. ACM. Paper 338 .

[63] Iain J Marshall, Joël Kuiper, and Byron C Wallace. 2016. RobotReviewer: evaluation of a system for automatically assessing bias in clinical trials. Fournal of the American Medical Informatics Association 23, 1 (January 2016), 193-201.

[64] Damien Masson, Sylvain Malacria, Edward Lank, and Géry Casiez. 2020. Chameleon: Bringing Interactivity to Static Digital Documents. In Proceedings of the CHI Conference on Human Factors in Computing Systems. ACM. Paper 432.

[65] MathJax. https://mathjax.org/. Last accessed January 8, 2021.

[66] Elisa Mattiello. 2017. Analogy in Word-Formation: A Study of English Neologisms and Occasionalisms. De Gruyter Mouton.

[67] Melissa McCartney, Chazman Childers, Rachael R. Baiduc, and Kitch Barnicle. 2018. Annotated Primary Literature: A Professional Development Opportunity in Science Communication for Graduate Students and Postdocs. Fournal of Microbiology \& Biology Education 19, 1 (March 2018), 1-13.

[68] MediaWiki contributors. Page Previews. https://www.mediawiki.org/wiki/ Page_Previews. Last accessed September 16, 2020.

[69] Mozilla and individual contributors. pdf.js. https://mozilla.github.io/pdf.js/. Last accessed September 16, 2020.

[70] David Nicholas, Peter Williams, Ian Rowlands, and Hamid R. Jamali. 2010. Researchers' e-journal use and information seeking behaviour. fournal of Information Science 36, 4 (2010), 494-516.

[71] Don Norman. 2013. The design of everyday things. Basic Books. See pages 288-291, section "The Future of Books".

[72] Kenton O'Hara. 1996. Towards a Typology of Reading Goals. Technical Report. Rank Xerox Research Centre.

[73] Robert Pagel and Moritz Schubotz. 2014. Mathematical Language Processing Project. In Proceedings of the Conference on Intelligent Computer Mathematics.

[74] PeerLibrary. https://peerlibrary.org/. Last accessed September 16, 2020.

[75] Bui Hai Phong, Thang Manh Hoang, and Thi-Lan Le. 2020. A Hybrid Method for Mathematical Expression Detection in Scientific Document Images. IEEE Access 8 (2020), 83663-83684.

[76] Peter Pirolli and Stuart K. Card. 1999. Information Foraging. Psychological Review 106, 4 (1999), 643-675.

[77] Antoine Ponsard, Francisco Escalona, and Tamara Munzner. 2016. PaperQuest: A Visualization Tool to Support Literature Review. In Proceedings of the CHI Conference on Human Factors in Computing Systems. ACM, 2264-2271.

[78] Brett Powley, Robert Dale, and Ilya Anisimoff. 2009. Enriching a Document Collection by Integrating Information Extraction and PDF Annotation. In Document Recognition and Retrieval. International Society for Optics and Photonics.

[79] PubMed. https://www.ncbi.nlm.nih.gov/pmc/articles/PMC4765694/. Example document; Last accessed September 16, 2020

[80] Xin Qian, Matt J. Erhart, Aniket Kittur, Wayne G. Lutters, and Joel Chan. 2019. Beyond iTunes for Papers: Redefining the Unit of Interaction in Literature Review Tools. In Proceedings of the Conference on Computer-Supported Cooperative Work and Social Computing. ACM, 341-346.

[81] Warren B. Roby. 1999. "What's in a gloss?": A commentary on Lara L. Lomicka's "To gloss or not to gloss": An investigation of reading comprehension online. Language Learning \& Technology 2, 2 (January 1999), 94-101.

[82] Susanne Rott. 2007. The Effect of Frequency of Input-Enhancements on Word Learning and Text Comprehension. Language Learning 57, 2 (June 2007), 165199.

[83] Somali Roy. 2014. Evaluating novel pedagogy in higher education: A case study of e-Proofs. Ph.D. Dissertation. Loughborough University.

[84] Somali Roy, Matthew Inglis, and Lara Alcock. 2017. Multimedia resources designed to support learning from written proofs: An eye-movement study. Educational Studies in Mathematics 96, 2 (2017), 249-266.

[85] Nipun Sadvilkar and Mark Neumann. 2020. PySBD: Pragmatic Sentence Boundary Disambiguation. In Proceedings of the Second Workshop for NLP Open Source Software. Association for Computational Linguistics, 110-114.

[86] Bill N. Schilit, Gene Golovchinsky, and Morgan N. Price. 1998. Beyond Paper: Supporting Active Reading with Free Form Digital Ink Annotations. In Proceedings of the CHI Conference on Human Factors in Computing Systems. ACM, 249-256.

[87] Scholarcy. https://www.scholarcy.com/scholarcy-features/. Last accessed July $24,2020$.

[88] Ariel S. Schwartz and Marti A. Hearst. 2003. A simple algorithm for identifying abbreviation definitions in biomedical text. In Pacific Symposium on Biocomputing. World Scientific Press, 451-462.

[89] ScienceDirect. https://sciencedirect.com/science/article/pii/S0939388918301181. Example document; Last accessed September 16, 2020.

[90] Mary D. Shepherd and Carla C. Van De Sande. 2014. Reading mathematics for understanding-From novice to expert. The fournal of Mathematical Behavior 35 (September 2014), 74-86.

[91] Noah Siegel, Nicholas Lourie, Russell Power, and Waleed Ammar. 2018. Extracting Scientific Figures with Distantly Supervised Neural Networks. In Proceedings of the foint Conference on Digital Libraries. ACM, 223-232. 
[92] Springer. https://link.springer.com/chapter/10.1007/978-3-030-01424-7_27. Example document; Last accessed September 16, 2020

[93] Emma Strubell, Patrick Verga, Daniel Andor, David Weiss, and Andrew McCallum. 2018. Linguistically-Informed Self-Attention for Semantic Role Labeling. (2018). arXiv:1804.08199 [cs.CL]

[94] Craig Tashman and W. Keith Edwards. 2011. Active Reading and Its Discontents: The Situations, Problems and Ideas of Readers. In Proceedings of the CHI Conference on Human Factors in Computing Systems. ACM, 2927-2936.

[95] Craig S. Tashman and W. Keith Edwards. 2011. LiquidText: A Flexible, Multitouch Environment to Support Active Reading. In Proceedings of the CHI Conference on Human Factors in Computing Systems. ACM, 3285-3294.

[96] Alan Taylor. 2006. The Effects of CALL versus Traditional L1 Glosses on L2 Reading Comprehension. CALICO journal 23, 2 (2006), 309-318.

[97] Carol Tenopir, Donald W. King, Sheri Edwards, and Lei Wu. 2009. Electronic journals and changes in scholarly article seeking and reading patterns. 61,1 (2009), 5-32

[98] Carol Tenopir, Elina Late, Sanna Talja, and Lisa Christian. 2019. Changes in Scholarly Reading in Finland Over a Decade: Influences of E-Journals and Social Media. Libri 69, 3 (2019), 169-187.

[99] Amir Pouran Ben Veyseh, Franck Dernoncourt, Thien Huu Nguyen, Walter Chang, and Leo Anthony Celi. 2020. Acronym Identification and Dis ambiguation Shared Tasks for Scientific Document Understanding. (2020). arXiv:2012.11760 [cs.CL]

[100] VSCode. https://code.visualstudio.com/. Last accessed September 16, 2020.

[101] Keith Weber. 2008. How Mathematicians Determine if an Argument Is a Valid Proof. Fournal for Research in Mathematics Education 39, 4 (July 2008), 431-459.

[102] H. G. Widdowson. 1978. Teaching Language as Communication. Oxford University Press.

[103] Austin P. Wright, Zijie J. Wang, Haekyu Park, Grace Guo, Fabian Sperrle, Mennatallah El-Assady, Alex Endert, Daniel Keim, and Duen Horng (Polo) Chau. 2020. A Comparative Analysis of Industry Human-AI Interaction Guidelines. (2020). arXiv:2010.11761 [cs.HC]

[104] Akifumi Yanagisawa, Stuart Webb, and Takumi Uchihara. 2020. How do different forms of glossing contribute to L2 vocabulary learning from reading? A metaregression analysis. Studies in Second Language Acquisition 42, 2 (May 2020) 411-438.

[105] Ming Yin, Jennifer Wortman Vaughan, and Hanna Wallach. 2019. Understanding the Effect of Accuracy on Trust in Machine Learning Models. In Proceedings of the CHI Conference on Human Factors in Computing Systems. ACM. Paper 279

[106] Dongwook Yoon, Nicholas Chen, Francois Guimbretière, and Abigail Sellen. 2014. RichReview: Blending Ink, Speech, and Gesture to Support Collaborative Document Review. In Proceedings of the Symposium on User Interface Software and Technology. ACM, 481-490.

[107] Robert Zeleznik, Andrew Bragdon, Ferdi Adeputra, and Hsu-Sheng Ko. 2010. Hands-On Math: A page-based multi-touch and pen desktop for technical work and problem solving. In Proceedings of the Symposium on User Interface Software and Technology. ACM, 17-26.

[108] Polle T. Zellweger, Bay-Wei Chang, and Jock D. Mackinlay. 1998. Fluid Links for Informed and Incremental Link Transitions. In Proceedings of the Conference on Hypertext and Hypermedia. ACM, 50-57.

[109] Xiaolong Zhang, Yan Qu, C. Lee Giles, and Piyou Song. 2008. CiteSense: Supporting Sensemaking of Research Literature. In Proceedings of the CHI Conference on Human Factors in Computing Systems. ACM, 677-680.

[110] Tianchang Zhao and Kyusong Lee. 2020. Talk to Papers: Bringing Neural Question Answering to Academic Search. In Annual Meeting of the Association for Computational Linguistics. Association for Computational Linguistics, 30-36.

[111] John Zimmerman, Jodi Forlizzi, and Shelley Evenson. 2007. Research Through Design as a Method for Interaction Design Research in HCI. In Proceedings of the CHI Conference on Human Factors in Computing Systems. ACM, 493-502.

\section{A APPENDIX}

\section{A.1 Statistical Analysis}

A.1.1 Modeling Mixed-Effects in Repeated Measures Studies. For the analysis in Section 6, we used the generalized linear mixedeffects model (GLMM). GLMMs are often used to analyze repeated measures, in which the same subject contributes multiple (potentially correlated) measurements [58]. They have been used to analyze measurements from studies in medicine [16], the behavioral sciences [19], and human-computer interaction [32].
A.1.2 F-Tests for Significant Effect of Interface. For each of the quantitative measurements $(y)$, we fit a GLMM with fixed effects $\beta$ for the interface $\left(x_{1}\right)$ and question $\left(x_{2}\right)$ factors (and a fixed-effects interaction term). The models were fit using the LME4 package in $\mathrm{R}$ [6]. More precisely, we fit the following GLMM:

$$
g(E[y])=\beta_{0}+\gamma_{j}+\beta_{1} x_{1}+\beta_{2} x_{2}+\beta_{3} x_{1} x_{2},
$$

where $g$ is the link function, and the random intercepts $\gamma_{j} \sim$ $\mathcal{N}\left(0, \sigma_{\gamma}^{2}\right)$ capture individual variation of each participant $j$. For EASE, Confidence, Time, Distance, and Area, we used the identity link $g(z)=z$. For CORRECT, which we treated as a Bernoulli variable, we used the logit link $g(p)=\log (p /(1-p))$.

Using the LMERTEST R package [52], we conducted $F$-tests for differences in fixed-effect estimates between each interface option, repeated for each $y .{ }^{4}$ We performed Holm-Bonferroni [36] correction on the $p$-values using the P.ADJUST R package. We found significance for CoRrect $(p=.047)$, EASE $(p<.001)$, Confidence $(p=.040)$, Time $(p<.001)$, Distance $(p=.005)$, Area $(p<.001)-$ even while controlling for question and participant-specific effects. That is to say, for these metrics, the $F$-test has identified that the choice of interface (BASIC, Declutter, or ScholarPhi) is a significant factor. Note that the $F$-test does not assess which of these interfaces is more or less impactful on the metric.

A.1.3 Tests for Pairwise Differences in Mean Effects between Interfaces. We conducted a post-hoc analysis to quantify the pairwise differences in mean effects between interfaces on $y$ under the GLMM (and controlling for question). Two-sided $t$-tests for pairwise comparisons were computed using the EMMEANs R package, yielding the results shown in Table 2 .

Because the GLMM for $y=$ CORRECT was fit using a logit link, direct testing of pairwise comparisons $\hat{y}_{i}-\hat{y}_{j}=\hat{\operatorname{Pr}}$ (CORRECT $=$ $1 \mid i)-\hat{\operatorname{Pr}}($ Correct $=0 \mid j)$ was not possible. We used the transform option in EMMEANs to perform the tests on the log-odds $\log \operatorname{Pr}($ Conrect $=1) / \operatorname{Pr}($ Correct $=0)$ scale, which are linear under the GLMM, before applying the inverse-link $g^{-1}$ transformation to return to the probability $\operatorname{Pr}($ CORRECT $=1)$ scale. This yielded the estimated (absolute) differences in reported in Table 2.

A.1.4 Ordinal Regression for Likert-Scale Variables. As EASE and CONFIDENCE were measured on a 5-point Likert scale, a linear GLMM estimated means was seen as potentially ill-suited for analysis, especially if EASE and CONFIDENCE are not sufficiently normally distributed. We additionally performed likelihood ratio tests after fitting analogous cumulative link mixed-effects models (CLMM) provided in the ORDINAL $\mathrm{R}$ package [15]. Likelihood ratio tests, which are similar to $F$-tests but more conservative, yielded similar $p$-values-EAse $(p<.001)$ and CONFIDENCE $(p=0.045)-$ and resulted in the same conclusions as those when using the GLMM. Since pairwise comparisons are not available through EMMEANs (or other libraries) for CLMMs, we opted to use the GLMM model for EASE and CONFIDENCE to enable subsequent analysis for Table 2.

${ }^{4}$ The $F$-test is not applicable when $y \sim$ Bernoulli, so we performed the similar, but slightly more conservative, likelihood ratio test for $y=$ CORRECT [52]. 